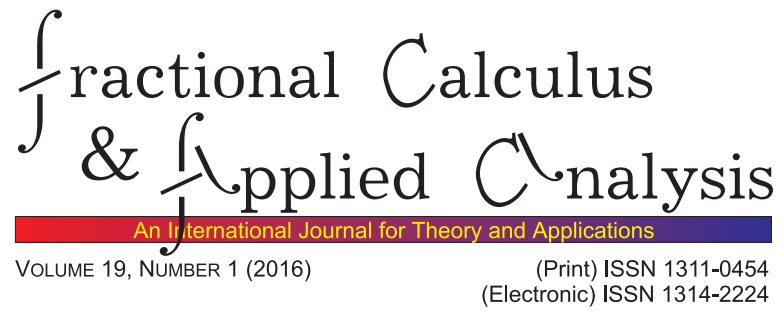

RESEARCH PAPER

\title{
ERROR ESTIMATES FOR APPROXIMATIONS OF DISTRIBUTED ORDER TIME FRACTIONAL DIFFUSION WITH NONSMOOTH DATA
}

\author{
Bangti Jin ${ }^{1}$, Raytcho Lazarov ${ }^{2}$, Dongwoo Sheen ${ }^{3}$, Zhi Zhou ${ }^{4}$
}

\begin{abstract}
In this work, we consider the numerical solution of a distributed order subdiffusion model, arising in the modeling of ultra-slow diffusion processes. We develop a space semidiscrete scheme based on the Galerkin finite element method, and establish error estimates optimal with respect to data regularity in $L^{2}(\Omega)$ and $H^{1}(\Omega)$ norms for both smooth and nonsmooth initial data. Further, we propose two fully discrete schemes, based on the Laplace transform and convolution quadrature generated by the backward Euler method, respectively, and provide optimal $L^{2}(\Omega)$ error estimates, which exhibits exponential convergence and first-order convergence in time, respectively. Extensive numerical experiments are provided to verify the error estimates for both smooth and nonsmooth initial data.
\end{abstract}

MSC 2010: Primary 65M60; Secondary 35R11, 65M15

Key Words and Phrases: distributed order, fractional diffusion, Galerkin finite element method, fully discrete scheme, error estimates

\section{Introduction}

We consider the following initial boundary value problem for a distributed order time fractional diffusion equation for $u(x, t)$ :

(c) 2016 Diogenes Co., Sofia

pp. 69-93, DOI: 10.1515/fca-2016-0005

DE GRUYTER 


$$
\begin{array}{rlrl}
\mathrm{D}_{t}^{[\mu]} u-\Delta u & =0 & & \text { in } \Omega \times(0, T], \\
u=0 & & \text { on } \partial \Omega \times(0, T], \\
u(0) & =v & & \text { in } \Omega,
\end{array}
$$

where $\Omega$ is a bounded convex polygonal domain in $\mathbb{R}^{d}(d=1,2,3)$ with a boundary $\partial \Omega, v$ is a given function on $\Omega$, and $T>0$ is a fixed value. Here, $\mathrm{D}_{t}^{[\mu]} u$ denotes the distributed order fractional derivative of $u$ in time $t$ (with respect to $\mu$ ) defined by

$$
\mathrm{D}_{t}^{[\mu]} u(t)=\int_{0}^{1} \partial_{t}^{\alpha} u(t) \mu(\alpha) d \alpha
$$

where $\partial_{t}^{\alpha} u, 0<\alpha<1$, is the Caputo derivative of order $\alpha$ in $t$, defined by [16, p. 91]

$$
\partial_{t}^{\alpha} u(t)=\frac{1}{\Gamma(1-\alpha)} \int_{0}^{t}(t-s)^{-\alpha} u^{\prime}(s) d s,
$$

where $\Gamma(\cdot)$ denotes Euler's Gamma function. In this paper we consider a weight function $\mu \in C[0,1]$ with $0 \leq \mu<1$ and $\mu(0) \mu(1)>0$.

In the last few decades, fractional calculus has been extensively studied and successfully employed to model subdiffusion, in which the mean squared variance grows slower than that in a Gaussian process. The subdiffusion model involves a Caputo derivative $\partial_{t}^{\alpha_{0}} u$ of order $\alpha_{0} \in(0,1)$ in $t$

$$
\partial_{t}^{\alpha_{0}} u-\Delta u=f \quad \text { in } \Omega \times(0, T]
$$

describes subdiffusion processes. The model (1.2) is recovered from (1.1) with a singular weight $\mu(\alpha)=\delta\left(\alpha-\alpha_{0}\right)$, the Dirac delta function at $\alpha_{0}$. Physically, subdiffusion can be characterized by a unique diffusion exponent known as the Hurst exponent [3]. In practice, the physical process may not possess a unique Hurst exponent, and the model (1.1) provides a flexible framework for describing a host of continuous and nonstationary signals [3, 4, 29]. It is often employed to describe ultraslow diffusion, where the mean squared variance grows only logarithmically with time.

In recent years, the theoretical study of problem (1.1) has attracted some attention. Kochubei [17 made some early contributions to the rigorous analysis of the model (1.1), by constructing fundamental solutions and establishing their positivity and subordination property. Mainardi et al. [22] studied the existence of a solution, asymptotic behavior, and positivity etc. Meerschaert and Scheffler [25] gave a stochastic model for ultraslow diffusion; see also [31. Luchko [21] showed a weak maximum principle for the problem. Li et al [18] established a sharp asymptotic behavior of the solution for $t \rightarrow 0$ and $t \rightarrow \infty$, in the case of continuous density $\mu$ with $\mu(1)>0$; see also [1] for further discussions. 
The solution to (1.1) is rarely available in closed form, which necessitates the development of efficient numerical schemes. Despite the extensive studies on (1.2), there are only very few studies [6, 15, 26, 9, 7] on (1.1). Diethelm and Ford [6] developed a numerical scheme for distributed order fractional ODEs. It approximates $\mathrm{D}_{t}^{[\mu]} u(t)$ by quadrature. This technique was also employed to solve nonlinear distributed-order fractional ODEs in [15, but without any analysis. Recently, Morgado and Rebelo [26] developed an implicit finite difference method for (1.1) with a Lipschitz nonlinear source term. The scheme approximates $\mathrm{D}_{t}^{[\mu]} u(t)$ by quadrature together with the backward finite difference in time, and the second-order finite difference in space. Its stability, and an error estimate $O\left(h^{2}+\tau+(\delta \alpha)^{2}\right)$ (with $h, \tau$ and $\delta \alpha$ being the mesh size, time step size and step size for quadrature rule, respectively) were established by assuming that the solution $u$ is $C^{2}$ in time and $C^{4}$ in space and $\mu(\alpha)$ is sufficiently regular. See also [9] a high-order difference scheme with $v \equiv 0$, and also [7]. The regularity requirement is restrictive. The development of numerical schemes for (1.1) with nonsmooth data and their rigorous analysis have not been carried out, despite its importance, e.g., in inverse and optimal control problems [14].

In this work, we develop a Galerkin finite element method (FEM) for problem (1.1). It is based on the finite element space $X_{h}$ of continuous piecewise linear functions over a family of shape regular quasi-uniform partitions $\left\{\mathcal{T}_{h}\right\}_{0<h<1}$ of $\Omega$ into $d$-simplexes, where $h$ is the mesh size. Then the space semidiscrete Galerkin FEM is given by: find $u_{h}(t) \in X_{h}$ such that

$$
\left(\mathrm{D}_{t}^{[\mu]} u_{h}, \chi\right)+a\left(u_{h}, \chi\right)=0, \quad \forall \chi \in X_{h}, T \geq t>0,
$$

with $u_{h}(0)=v_{h}$, where $(\cdot, \cdot)$ denotes the $L^{2}(\Omega)$-inner product, $a(u, w)=$ $(\nabla u, \nabla w)$ for $u, w \in H_{0}^{1}(\Omega)$, and $v_{h} \in X_{h}$ is an approximation of $v$. Further, we develop two fully discrete schemes based on the Laplace transform and convolution quadrature generated by the backward Euler method.

Our main contributions are as follows. First, in Theorem 2.1, we establish sharp regularity estimates for problem (1.1). Second, in Theorems 3.1 and 3.2, we derive optimal error estimates for the semidiscrete scheme (1.3). Third, we develop a fully discrete scheme based on the Laplace transform, using a contour representation of the semidiscrete solution with a hyperbolic contour and trapezoidal quadrature. We show its exponential convergence for a fixed time $t$, cf. Theorem 4.1. Last, we develop a second fully discrete scheme based on convolution quadrature, generated by the backward Euler method, and in Theorem [5.3, establish a first-order convergence. All these error estimates are nearly optimal and expressed in terms of the data regularity directly.

The model (1.1) is closely related to parabolic equations with a positive type memory term, for which there are many studies on numerical schemes 
based on convolution quadrature [19, 20, 5] and Laplace transform [27, 28, 32. These interesting works have inspired the current work. However, they do not cover (1.1), due to the general kernel function involved. Instead, we shall opt for a direct strategy by bounding the kernel function.

The rest of the paper is organized as follows. In Section 2, we recall the solution theory of the model (1.1). In Section 3, we develop a space semidiscrete Galerkin scheme. Two fully discrete schemes are given in Sections 4 and 5. Finally, to verify the theory, we present in Section 6 some numerical experiments. Throughout, the notation $c$, with or without a subscript, denotes a generic constant, which may differ at different occurrences, but it is always independent of the mesh size $h$, the number $N$ of quadrature points, and time step size $\tau$. Further, we denote by $\|\cdot\|$ the $L^{2}(\Omega)$ norm.

\section{Solution Theory}

In this part, we discuss the solution theory of problem (1.1). We denote by ${ }^{\wedge}$ the Laplace transform. Next we denote by $A$ the operator $-\Delta$ with a homogeneous Dirichlet boundary condition with a domain $D(A)=H_{0}^{1}(\Omega) \cap$ $H^{2}(\Omega)$. It is known that the operator $A$ generates a bounded analytic semigroup of angle $\pi / 2$, i.e., for any $\theta \in(\pi / 2, \pi)$ [10, p. 321, Prop. C.4.2]

$$
\left\|(z I+A)^{-1}\right\| \leq|\Im(z)|^{-1} \leq|z \sin (\theta)|^{-1} \quad \forall z \in \Sigma_{\theta}^{\prime},
$$

where $\Sigma_{\theta}^{\prime}$ is a sector with the origin excluded, i.e., $\Sigma_{\theta}=\{z \in \mathbb{C}:|\arg (z)|<$ $\theta\}, \Sigma_{\theta}^{\prime}=\Sigma_{\theta} \backslash\{0\}$. Then by (1.1) and the relation $\widehat{\partial_{t}^{\alpha} u}=z^{\alpha} \widehat{u}-z^{\alpha-1} u(0)$

$$
z w(z) \widehat{u}(z)+A \widehat{u}(z)=w(z) v,
$$

with $w(z)=\int_{0}^{1} z^{\alpha-1} \mu(\alpha) d \alpha$. Hence, $u(t)$ is represented by

$$
u(t)=S(t) v:=\frac{1}{2 \pi \mathrm{i}} \int_{\Gamma_{\theta, \delta}} e^{z t} H(z) v d z,
$$

where $H(z)=(z w(z) I+A)^{-1} w(z)$, and $\Gamma_{\theta, \delta}=\{z \in \mathbb{C}:|z|=\delta,|\arg z| \leq$ $\theta\} \cup\left\{z \in \mathbb{C}: z=\rho e^{ \pm i \theta}, \rho \geq \delta\right\}$.

Now we give a few properties of $w(z)$. The first is the sector-preserving property, since $z^{\alpha} \in \Sigma_{\theta}$ for any $\alpha \in(0,1)$ and $z \in \Sigma_{\theta}$.

Lemma 2.1. Let $\theta \in(\pi / 2, \pi)$. Then $z w(z) \in \Sigma_{\theta}$ for all $z \in \Sigma_{\theta}$.

The second result is an upper bound on $w(z)$.

Lemma 2.2. Let $\mu \in C[0,1]$ with $\mu \geq 0$. Then there holds

$$
|w(z)| \leq\|\mu\|_{C[0,1]}(|z|-1) /(|z| \ln |z|) .
$$

The third result gives a lower bound on $z w(z)$. 
Lemma 2.3. Let $\theta \in(\pi / 2, \pi)$ with $\mu(0) \mu(1)>0$. Then there exists a constant $c>0$ dependent only on $\theta$ and $\mu$ such that for any $z \in \Sigma_{\theta}^{\prime}$

$$
\begin{aligned}
& |z w(z)| \geq c(|z|-1) / \ln |z| \\
& |z| w(|z|) \geq|z w(z)| \geq c|z| w(|z|) .
\end{aligned}
$$

P r o o f. Let $z=r e^{\mathrm{i} \varphi}$. Using $\mu(1)>0$ and $\mu \in C[0,1]$, we can find a small $\epsilon_{1}>0$ such that $\min _{\alpha \in\left[1-\epsilon_{1}, 1\right]} \mu(\alpha) \geq \delta_{1}>0$. Thus for $r \geq 1$, there holds $\int_{0}^{1} r^{\alpha} \mu(\alpha) d \alpha \geq \int_{1-\epsilon_{1}}^{1} r^{\alpha} \mu(\alpha) d \alpha \geq \delta_{1} \int_{1-\epsilon_{1}}^{1} r^{\alpha} d \alpha \geq \epsilon_{1} \delta_{1} \int_{0}^{1} r^{\alpha} d \alpha$. Similarly, we may find a small $\epsilon_{2}>0$ such that $\min _{\alpha \in\left[0, \epsilon_{2}\right]} \mu(\alpha) \geq \delta_{2}>0$ and then for all $r<1$, there holds $\int_{0}^{1} r^{\alpha} \mu(\alpha) d \alpha \geq \epsilon_{2} \delta_{2} \int_{0}^{1} r^{\alpha} d \alpha$. Hence for $\varphi \in(\theta-\pi, \pi-\theta)$, we get for $c_{1}=\min \left(\epsilon_{1} \delta_{1}, \epsilon_{2} \delta_{2}\right)$

$$
|z w(z)| \geq \Re(z w(z)) \geq \cos (\pi-\theta) \int_{0}^{1} r^{\alpha} \mu(\alpha) d \alpha \geq c_{1} \cos (\pi-\theta) \int_{0}^{1} r^{\alpha} d \alpha .
$$

It suffices to consider $\varphi \in[\pi-\theta, \theta]$. Since $\mu(0)>0$, there exists a small $\epsilon_{0}>0$ such that $\min _{\alpha \in\left[0, \epsilon_{0}\right]} \cos (\alpha \pi) \mu(\alpha)=\delta_{0}>0$, and thus

$$
\begin{aligned}
\Re(z w(z)) & \geq \int_{0}^{\epsilon_{0}} r^{\alpha} \cos (\alpha \varphi) \mu(\alpha) d \alpha-\int_{\epsilon_{0}}^{1} r^{\alpha}|\cos (\alpha \varphi)| \mu(\alpha) d \alpha \\
& \geq \delta_{0} \int_{0}^{\epsilon_{0}} r^{\alpha} d \alpha-\|\mu\|_{C[0,1]} \int_{\epsilon_{0}}^{1} r^{\alpha} d \alpha \\
& \geq-(\ln r)^{-1}\left[\delta_{0}-r^{\epsilon_{0}}\left(\delta_{0}+\|\mu\|_{C[0,1]}\right)\right] .
\end{aligned}
$$

Consequently,

$|z w(z)| \geq \Re(z w(z)) \geq \frac{\delta_{0}}{2} \int_{0}^{1} r^{\alpha} d \alpha \quad \forall r \leq r_{0}=:\left(\delta_{0} /\left(2\left(\delta_{0}+\|\mu\|_{C[0,1]}\right)\right)\right)^{1 / \epsilon_{0}}$.

Similarly, (2.3) holds for $r \geq r_{0}$ and $\varphi \in[\pi-\theta, \theta]$, thereby showing (2.3). The estimate (2.4) follows from $\|\mu\|_{C[0,1]} \int_{0}^{1} r^{\alpha} d \alpha \geq \int_{0}^{1} r^{\alpha} \mu(\alpha) d \alpha=$ $|z| w(|z|)$ and the inequality $|z w(z)| \leq|z| w(|z|)$.

Now we give the stability of problem (1.1).

Theorem 2.1. Let $\mu \in C[0,1]$ with $\mu \geq 0$ and $\mu(0) \mu(1)>0$. Then the solution $u$ to problem (1.1) satisfies for $t \in(0, T]$ and $\nu=0,1$ :

$$
\begin{gathered}
\left\|A^{\nu} S^{(m)}(t) v\right\| \leq c_{T} t^{-m-\nu} \ell_{1}(t)^{\nu}\|v\|, v \in L^{2}(\Omega), m \geq 0 \\
\left\|A^{\nu} S^{(m)}(t) v\right\| \leq c t^{-m+1-\nu} \ell_{2}(t)^{1-\nu}\|A v\|, v \in D(A), \nu+m \geq 1
\end{gathered}
$$

where $\ell_{1}(t)=(\ln (2 T / t))^{-1}, \ell_{2}(t)=\ln \left(\max \left(t^{-1}, 2\right)\right)$ and $c_{T}>0$ may depend on $d, \Omega, \mu, M, m$ and $T$. 
P r o o f. The existence and uniqueness of a weak solution was shown in [18], and it suffices to show (2.5) and (2.6). First, by (2.1), we have $\|H(z)\|=\left\|(z w(z) I+A)^{-1}\right\||w(z)| \leq M /|z|$ for all $z \in \Sigma_{\theta}^{\prime}$. Let $t>0$, $\theta \in(\pi / 2, \pi), \delta>0$. We choose $\delta=t^{-1}$ and denote for short $\Gamma=\Gamma_{\theta, \delta}$. First we derive (2.5) for $\nu=0$ and $m \geq 0$. By (2.2), we deduce

$$
\begin{aligned}
\left\|S^{(m)}(t)\right\| & =\left\|\frac{1}{2 \pi \mathrm{i}} \int_{\Gamma} z^{m} e^{z t} H(z) d z\right\| \leq c \int_{\Gamma}|z|^{m} e^{\Re(z) t}\|H(z)\||d z| \\
& \leq c\left(\int_{t^{-1}}^{\infty} r^{m-1} e^{r t \cos \theta} d r+\int_{-\theta}^{\theta} e^{\cos \psi} t^{-m} d \psi\right) \leq c t^{-m} .
\end{aligned}
$$

Next we prove (2.5) for $\nu=1$ and $m \geq 0$, by taking $\delta=2 T / t$ in $\Gamma$. By applying $A$ to (2.2) and differentiating with respect to $t$ we arrive at

$$
A S^{(m)}(t)=\frac{1}{2 \pi \mathrm{i}} \int_{\Gamma} z^{m} e^{z t} A H(z) d z .
$$

Since $A H(z)=A(z w(z) I+A)^{-1} w(z)=(I-z H(z)) w(z)$, Lemma 2.2 yields $\|A H(z)\| \leq c|w(z)| \leq c(|z|-1) /(|z| \ln |z|)$ for all $z \in \Sigma_{\theta}^{\prime}$. Hence (2.7) gives

$$
\begin{aligned}
\left\|A S^{(m)}(t)\right\| \leq & c \int_{\Gamma}|z|^{m} \frac{|z|-1}{|z| \ln |z|} e^{\Re(z) t}|d z| \leq c \int_{\delta}^{\infty} r^{m-1} \frac{r-1}{\ln r} e^{r t \cos \theta} d r \\
& +c_{T} t^{-m} \frac{\delta-1}{\ln \delta} \int_{-\theta}^{\theta} e^{2 T \cos \psi} d \psi=: I+I I .
\end{aligned}
$$

Since $\delta \geq 2$, we can bound the first term $I$ by

$$
I \leq c \int_{\delta}^{\infty} r^{m}(\ln r)^{-1} e^{r t \cos \theta} d r \leq c \ell_{1}(t) \int_{\delta}^{\infty} r^{m} e^{r t \cos \theta} d r \leq c_{T} t^{-m-1} \ell_{1}(t) .
$$

The second term $I I$ is bounded by $I I=c_{T} t^{-m}(\delta-1) / \ln \delta \leq c_{T} t^{-m-1} \ell_{1}(t)$. This shows (2.5). To prove (2.6) with $\nu=0$, we choose $\delta=t^{-1}$. Then,

$$
S^{(m)}(t) v=\frac{1}{2 \pi \mathrm{i}} \int_{\Gamma} z^{m} e^{z t} H(z) v d z=\frac{1}{2 \pi \mathrm{i}} \int_{\Gamma} z^{m-1} e^{z t} z A^{-1} H(z) A v d z .
$$

Since $z A^{-1} H(z)=A^{-1}-(z w(z) I+A)^{-1}$ and $\int_{\Gamma} z^{m-1} e^{z t} d z=0$ for $m \geq 1$, we have

$$
\begin{aligned}
S^{(m)}(t) v & =\frac{1}{2 \pi \mathrm{i}} \int_{\Gamma} z^{m-1} e^{z t} v d z-\frac{1}{2 \pi \mathrm{i}} \int_{\Gamma} z^{m-1} e^{z t}(z w(z) I+A)^{-1} d z A v \\
& =-\frac{1}{2 \pi \mathrm{i}} \int_{\Gamma} z^{m-1} e^{z t}(z w(z) I+A)^{-1} d z A v .
\end{aligned}
$$

By (2.1) and Lemma 2.3 we obtain $\left\|(z w(z) I+A)^{-1}\right\| \leq M|z w(z)|^{-1} \leq$ $c(|z|-1)^{-1} \ln |z|$. This and the monotonicity of $f(x)=-\ln (x) /(1-x)$ on the positive real axis $\mathbb{R}^{+}$yield 


$$
\begin{aligned}
&\left\|S^{(m)}(t) v\right\| \leq c\left(\int_{\Gamma}|z|^{m-1} e^{\Re(z) t}\left\|(z w(z) I+A)^{-1}\right\||d z|\right)\|A v\| \\
& \leq c\left(\int_{t^{-1}}^{\infty} e^{r t \cos \theta} r^{m-1} \frac{\ln r}{r-1} d r+t^{-m} \frac{\ln t^{-1}}{t^{-1}-1} \int_{-\theta}^{\theta} e^{\cos \psi} d \psi\right)\|A v\| \\
& \leq c t^{-m+1} \ln \left(t^{-1}\right)(1-t)^{-1}\|A v\| .
\end{aligned}
$$

For $t^{-1} \geq 2, \ln \left(t^{-1}\right) /(1-t) \leq 2 \ln \left(t^{-1}\right)$, and for $t^{-1}<2, \ln \left(t^{-1}\right) /(1-t)=$ $\ln (t) /(t-1) \leq 2 \ln (2)$. Thus $\left\|S^{(m)}(t) v\right\| \leq c t^{-m+1} \ell_{2}(t)\|A v\|$. Last, (2.6) with $\nu=1$ is equivalent to (2.5) with $\nu=0$ and $v$ by $A v$.

\section{Semidiscrete Discretization by Galerkin FEM}

Now we discuss the space semidiscrete scheme (1.3) based on the Galerkin FEM. On the space $X_{h}$, we define the $L^{2}(\Omega)$ projection $P_{h}: L^{2}(\Omega) \rightarrow X_{h}$ and the Ritz projection $R_{h}: H_{0}^{1}(\Omega) \rightarrow X_{h}$, respectively, by

$$
\begin{aligned}
\left(P_{h} \varphi, \chi\right) & =(\varphi, \chi) \quad \forall \chi \in X_{h}, \\
\left(\nabla R_{h} \varphi, \nabla \chi\right) & =(\nabla \varphi, \nabla \chi) \quad \forall \chi \in X_{h} .
\end{aligned}
$$

With the discrete Laplacian $\Delta_{h}: X_{h} \rightarrow X_{h}$ defined by $-\left(\Delta_{h} \varphi, \chi\right)=$ $(\nabla \varphi, \nabla \chi)$ for all $\varphi, \chi \in X_{h}$, and $A_{h}=-\Delta_{h}$, (1.3) can be rewritten as

$$
\mathrm{D}_{t}^{[\mu]} u_{h}(t)+A_{h} u_{h}(t)=0, t>0
$$

with $u_{h}(0)=v_{h} \in X_{h}$. For the error analysis, we employ an operator trick [8]. To this end, we first represent $u_{h}$ by

$$
u_{h}(t)=S_{h}(t) v_{h}:=\frac{1}{2 \pi \mathrm{i}} \int_{\Gamma_{\theta, \delta}} e^{z t}\left(z w(z) I+A_{h}\right)^{-1} w(z) v_{h} d z .
$$

Now we introduce the error function $e(t):=u(t)-u_{h}(t)$, which, by (2.2) and (3.2), is given by

$$
e(t)=\frac{1}{2 \pi \mathrm{i}} \int_{\Gamma_{\theta, \delta}} e^{z t} w(z)\left(\widehat{\varphi}(z)-\widehat{\varphi}_{h}(z)\right) d z,
$$

with $\widehat{\varphi}(z)=(z w(z) I+A)^{-1} v$ and $\widehat{\varphi}_{h}(z)=\left(z w(z) I+A_{h}\right)^{-1} P_{h} v$. The next lemma shows a bound on $\widehat{\varphi}_{h}-\widehat{\varphi}$. It follows from Lemma 2.1, similar to [2, Lemmas 3.3 and 3.4], and hence the proof is omitted.

Lemma 3.1. Let $v \in L^{2}(\Omega), z \in \Sigma_{\theta}$ with $\theta \in(\pi / 2, \pi), \widehat{\varphi}(z)=$ $(z w(z) I+A)^{-1} v$ and $\widehat{\varphi}_{h}(z)=\left(z w(z) I+A_{h}\right)^{-1} P_{h} v$. Then there holds

$$
\left\|\widehat{\varphi}(z)-\widehat{\varphi}_{h}(z)\right\|+h\left\|\nabla\left(\widehat{\varphi}(z)-\widehat{\varphi}_{h}(z)\right)\right\| \leq c h^{2}\|v\| .
$$

Now we can state an error estimate for nonsmooth data $v \in L^{2}(\Omega)$. 
Theorem 3.1. Let $u$ and $u_{h}$ be the solutions of problem (1.1) and (3.1) with $v \in L^{2}(\Omega)$ and $v_{h}=P_{h} v$, respectively. Then for $t>0$ and $\ell_{1}(t)=\ln (2 T / t)^{-1}$, there holds

$$
\left\|u(t)-u_{h}(t)\right\|+h\left\|\nabla\left(u(t)-u_{h}(t)\right)\right\| \leq c_{T} h^{2} t^{-1} \ell_{1}(t)\|v\| .
$$

P r o o f. By (3.3), with $\delta=2 T / t$ in $\Gamma_{\theta, \delta}$, and Lemmas 3.1 and 2.2 ,

$$
\|\nabla e(t)\| \leq \operatorname{ch} \int_{\delta}^{\infty} e^{r t \cos \theta} \frac{r-1}{r \ln r} d r\|v\|+\operatorname{ch} \int_{-\theta}^{\theta} e^{2 T \cos \psi} \frac{\delta-1}{\ln \delta} d \psi\|v\|:=I+I I .
$$

Now the first term $I$ can be bounded by

$$
I \leq c h \int_{\delta}^{\infty} \frac{e^{r t \cos \theta}}{\ln r} d r\|v\| \leq \frac{c h}{\ln \delta} \int_{\delta}^{\infty} e^{r t \cos \theta} d r\|v\| \leq c_{T} h t^{-1} \ell_{1}(t)\|v\|,
$$

and the second term $I I$ by

$$
I I \leq \frac{c_{T} h}{t \ln \delta} \int_{-\theta}^{\theta} e^{2 T \cos \psi} d \psi\|v\| \leq c_{T} h t^{-1} \ell_{1}(t)\|v\| .
$$

Thus the bound on $\|\nabla e(t)\|$ follows. The $L^{2}$ estimate is similar.

Next we turn to the case of smooth data $A v \in L^{2}(\Omega)$.

THEOREM 3.2. Let $u$ and $u_{h}$ be the solutions of problem (1.1) and (3.1) with $v \in D(A)$ and $v_{h}=R_{h} v$, respectively. Then for $t>0$,

$$
\left\|u(t)-u_{h}(t)\right\|+h\left\|\nabla\left(u(t)-u_{h}(t)\right)\right\| \leq c h^{2}\|A v\| .
$$

P r o o f. Like before, we take $\theta \in(\pi / 2, \pi)$ and $\delta=1 / t$ in the contour $\Gamma_{\theta, \delta}$. Then the error $e_{h}(t)=u(t)-u_{h}(t)$ can be represented by

$$
e_{h}(t)=\frac{1}{2 \pi \mathrm{i}} \int_{\Gamma_{\theta, \delta}} e^{z t} w(z)\left((z w(z) I+A)^{-1}-\left(z w(z) I+A_{h}\right)^{-1} R_{h}\right) v d z .
$$

Using $w(z)(z w(z) I+A)^{-1}=z^{-1} I-z^{-1}(z w(z) I+A)^{-1} A$, we deduce

$$
e_{h}(t)=\frac{1}{2 \pi \mathrm{i}}\left(\int_{\Gamma_{\theta, \delta}} e^{z t} z^{-1}\left(\widehat{\varphi}_{h}(z)-\widehat{\varphi}(z)\right) d z+\int_{\Gamma_{\theta, \delta}} e^{z t} z^{-1}\left(v-R_{h} v\right) d z\right),
$$

where $\widehat{\varphi}(z)=(z w(z) I+A)^{-1} A v$ and $\widehat{\varphi}_{h}(z)=\left(z w(z) I+A_{h}\right)^{-1} A_{h} R_{h} v$. Then Lemma 3.1 and the identity $A_{h} R_{h}=P_{h} A$ give

$$
\left\|\widehat{\varphi}(z)-\widehat{\varphi}_{h}(z)\right\|+h\left\|\nabla\left(\widehat{\varphi}(z)-\widehat{\varphi}_{h}(z)\right)\right\| \leq c h^{2}\|A v\| .
$$

Now it follows that

$$
\left\|e_{h}(t)\right\| \leq c h^{2}\|A v\|\left(\int_{t^{-1}}^{\infty} e^{r t \cos \theta} r^{-1} d r+\int_{-\theta}^{\theta} e^{\cos \psi} d \psi\right) \leq c h^{2}\|A v\|,
$$

which gives the $L^{2}$-estimate. The $H^{1}$ estimate is analogous. 
REMARK 3.1. The estimate for $v \in L^{2}(\Omega)$ deteriorates like $t^{-1} \ell_{1}(t)$ as $t \rightarrow 0^{+}$, which agrees with Theorem 2.1. The factor $t^{-1} \ell_{1}(t)$ is different from that for subdiffusion [12] and multi-term counterpart [11. For $A v \in$ $L^{2}(\Omega)$, the estimate is uniform in time.

\section{Fully Discrete Scheme I: Laplace Transform}

The first fully discrete scheme is based on Laplace transform, by applying a quadrature rule to the representation (3.2). We follow [24, 27, 28, 32] and deform the contour $\Gamma_{\theta, \delta}$ to be a curve with a parametric representation

$$
z(\xi):=\lambda(1+\sin (\mathrm{i} \xi-\psi))
$$

with $\lambda>0, \psi \in(0, \pi / 2)$ and $\xi \in \mathbb{R}$. The optimal choices of $\lambda$ and $\psi$ will be given in Lemma 4.4. This deformation is valid since it does not transverse the poles of $H(z) v=\left(z w(z)+A_{h}\right)^{-1} w(z) v$, cf., Lemmas 2.1 and 4.3. With $z=x+\mathrm{i} y$, the contour (4.1) is the left branch of the hyperbola

$$
((x-\lambda) /(\lambda \sin \psi))^{2}-(y /(\lambda \cos \psi))^{2}=1,
$$

which intersects the real axis at $x=\lambda(1 \pm \sin \psi)$ and has asymptotes $y= \pm(\lambda-x) \cot \psi$. Now we can represent $u_{h}(t)$ by

$$
u_{h}(t)=\int_{-\infty}^{\infty} \widehat{g}(\xi, t) d \xi
$$

with the integrand $\widehat{g}(\xi, t)$ being defined by

$$
\widehat{g}(\xi, t)=(2 \pi \mathrm{i})^{-1} e^{z(\xi) t}\left(z(\xi) w(z(\xi)) I+A_{h}\right)^{-1} w(z(\xi)) z^{\prime}(\xi) v_{h} .
$$

Now we describe the quadrature approximation. By setting $z_{j}=z\left(\xi_{j}\right)$ and $z_{j}^{\prime}:=z^{\prime}\left(\xi_{j}\right)$ with $\xi_{j}=j k$ and $k$ being the step size, we have

$$
U_{h}(t)=\frac{k}{2 \pi \mathrm{i}} \sum_{j=-\infty}^{\infty} e^{z_{j} t} \widehat{\phi}_{j} z_{j}^{\prime},
$$

and the truncated quadrature approximation

$$
U_{N, h}(t)=\frac{k}{2 \pi \mathrm{i}} \sum_{j=-N}^{N} e^{z_{j} t} \widehat{\phi}_{j} z_{j}^{\prime},
$$

with $\widehat{\phi}_{j}=\left(z_{j} w\left(z_{j}\right) I+A_{h}\right)^{-1} w\left(z_{j}\right) v_{h}$. To compute $U_{N, h}(t)$, we need to solve only $N+1$ elliptic problems, instead of $2 N+1$, by exploiting conjugacy: $z_{-j}=\overline{z_{j}}, w\left(z_{-j}\right)=\overline{w\left(z_{-j}\right)}, \widehat{\phi}_{-j}=\overline{\widehat{\phi}_{j}}, j=1, \cdots, N$. Indeed, since $z_{j}^{\prime}=$ $z^{\prime}\left(\xi_{j}\right)=\mathrm{i} \lambda \cos \left(\mathrm{i} \xi_{j}-\psi\right)$, with $\zeta_{j}=\lambda \cos \left(\mathrm{i} \xi_{j}-\psi\right)$, (4.4) is reduced to

$$
U_{N, h}(t)=\frac{k}{2 \pi} e^{z_{0} t} \widehat{\phi}_{0} \zeta_{0}+\frac{k}{\pi} \sum_{j=1}^{N} \Re\left\{e^{z_{j} t} \widehat{\phi}_{j} \zeta_{j}\right\} .
$$

Hence we solve the following complex-valued elliptic problems

$$
\left(z_{j} w\left(z_{j}\right) I+A_{h}\right) \widehat{\phi}_{j}=w\left(z_{j}\right) v_{h}, \quad j=0, \ldots, N .
$$


Next we define a strip by $\mathcal{S}_{a, b}=\{p=\xi+\mathrm{i} \eta: \xi \in \mathbb{R}, \eta \in(-b, a)\}$. The following lemma gives the quadrature error [23] [32, Theorem 2.1].

Lemma 4.1. Let $g$ be an analytic function in a strip $\mathcal{S}_{a, b}$ for some $a, b>0$, and $I$ and $I_{k}$, for $k>0$, be defined by $I=\int_{-\infty}^{\infty} g(x) d x$ and $I_{k}=k \sum_{j=-\infty}^{\infty} g(j k)$, respectively. Furthermore, assume that $g(z) \rightarrow 0$ uniformly as $|z| \rightarrow \infty$ in $\mathcal{S}_{a, b}$, and that there exist $M_{+}, M_{-}>0$ such that

$$
\lim _{r \rightarrow a^{-}} \int_{-\infty}^{\infty}|g(x+\mathrm{i} r)| d x \leq M_{+} \quad \text { and } \quad \lim _{s \rightarrow b^{-}} \int_{-\infty}^{\infty}|g(x-\mathrm{i} s)| d x \leq M_{-} .
$$

Then with $E^{+}=M_{+} /\left(e^{2 a \pi / k}-1\right)$ and $E^{-}=M_{-} /\left(e^{2 b \pi / k}-1\right)$, there holds

$$
\left|I-I_{k}\right| \leq E^{+}+E^{-} \text {. }
$$

The next lemma gives one crucial estimate on the map $z(p)$ over the strip $\mathcal{S}_{a, b}$. Even though the hyperbolic contour (4.1) has been extensively used, the estimate on the map $z(p)$ below seems to be new.

Lemma 4.2. Let $p=\xi+\mathrm{i} \eta$ with $\xi, \eta \in \mathbb{R}$. Then with $a=\pi / 2-\psi-\epsilon$ and $b=\psi-\epsilon$, for small $\epsilon>0$, there holds

$$
\begin{aligned}
& z(p) \in \Sigma_{\pi-\psi} \quad \text { and } \quad\left|z^{\prime}(p)\right||z(p)|^{-1} \leq c \epsilon^{-1} \quad \forall p \in \overline{\mathcal{S}}_{a, 0}, \\
& z(p) \in \Sigma_{\pi-\epsilon} \quad \text { and } \quad\left|z^{\prime}(p)\right||z(p)|^{-1} \leq c \quad \forall p \in \overline{\mathcal{S}}_{0, b} .
\end{aligned}
$$

P r o o f. For $p=\xi+\mathrm{i} \eta$ with $\xi, \eta \in \mathbb{R}$, then the image $z(p)$ in the parameterization (4.1) is given by $z(p)=\lambda(1-\sin (\psi+\eta) \cosh (\xi))+\mathrm{i} \lambda \cos (\psi+$ $\eta) \sinh (\xi)$, and its derivative $z^{\prime}(p)$ is given by $z^{\prime}(p)=\lambda \cosh \xi \cos (\psi+\eta)-$ $\mathrm{i} \sinh \xi \sin (\psi+\eta)$. By writing $z=x+\mathrm{i} y$, it can be expressed as the left branch of the hyperbola $((x-\lambda) /(\lambda \sin (\psi+\eta)))^{2}-(y /(\lambda \cos (\psi+\eta)))^{2}=1$. It intersects the real axis at $x=\lambda(1-\sin (\psi+\eta))$ and has the asymptotes $y= \pm(x-\lambda) \cot (\psi+\eta)$. Next we show (4.5) and (4.6). First, for $p \in \overline{\mathcal{S}}_{a, 0}$, i.e., $\eta \in[0, a], z(p)$ lies in the sector $\Sigma_{\pi-\psi}$. Since $\varphi:=\eta+\psi \in(\psi, \pi / 2-\epsilon)$, and $\sin (\pi / 2-\epsilon) \sim 1-\epsilon^{2} / 2 \leq 1-\epsilon^{2} / 3$ for small $\epsilon$, we have for all $\xi \in \mathbb{R}$

$$
\begin{aligned}
& \left|\frac{z^{\prime}(p)}{z(p)}\right|^{2}=\left|\frac{\cos (\varphi) \cosh (\xi)-\mathrm{i} \sin (\varphi) \sinh (\xi)}{(1-\cosh (\xi) \sin (\varphi))+\mathrm{i} \sinh (\xi) \cos (\varphi)}\right|^{2} \\
& =\frac{\cosh ^{2}(\xi)-\sin ^{2}(\varphi)}{(\cosh (\xi)-\sin (\varphi))^{2}}=\frac{\cosh (\xi)+\sin (\varphi)}{\cosh (\xi)-\sin (\varphi)} \leq \frac{1+\sin (\varphi)}{1-\sin (\varphi)} \\
& \leq \frac{2}{1-\left(1-\epsilon^{2} / 3\right)}=6 \epsilon^{-2} .
\end{aligned}
$$

Hence (4.5) holds true. For $p \in \overline{\mathcal{S}}_{0, b}$, i.e., $\eta \in[-b, 0], z(p)$ lies in $\Sigma_{\pi-(\eta+\psi)}$ $\subset \Sigma_{\pi-\epsilon}$. Further, since $\varphi:=\eta+\psi \in(\epsilon, \psi)$, for all $\xi \in \mathbb{R}$ 


$$
\left|z^{\prime}(p)\right|^{2}|z(p)|^{-2} \leq(1+\sin (\varphi)) /(1-\sin (\varphi)) \leq(1+\sin (\psi)) /(1-\sin (\psi)) .
$$

Then the desired result (4.6) follows directly.

The next result gives analyticity of and an estimate on $\widehat{g}(\xi, t)$ on $\mathcal{S}_{a, b}$.

LEMmA 4.3. Let $p=\xi+$ i $\eta$ with $\xi, \eta \in \mathbb{R}, a$ and $b$ be defined as in Lemma 4.2, and $\widehat{g}(p, t)$ be defined by (4.3). Then $\widehat{g}(p, t)$ is analytic on the strip $\mathcal{S}_{a, b}$, and there holds

$$
\|\widehat{g}(p, t)\| \leq c \epsilon^{-1} e^{\lambda(1-\sin (\psi+\eta) \cosh (\xi)) t}\left\|v_{h}\right\| \quad \forall p \in \mathcal{S}_{a, b} .
$$

P r o o f. For $p=\xi+\mathrm{i} \eta$ with $\xi, \eta \in \mathbb{R}, z(p)$ in (4.1) is given by

$$
z(p)=\lambda(1-\sin (\psi+\eta) \cosh (\xi))+\mathrm{i} \lambda \cos (\psi+\eta) \sinh (\xi) .
$$

By Lemmas 4.2 and 2.1 $z(p) w(z(p)) \in \Sigma_{\pi-\epsilon}$, and thus the function

$$
\widehat{g}(p, t)=(2 \pi \mathrm{i})^{-1} e^{z(p) t}\left(z(p) w(z(p)) I+A_{h}\right)^{-1} w(z(p)) z^{\prime}(p) v_{h}
$$

is analytic in $\mathcal{S}_{a, b}$. It suffices to show (4.7). For $p \in \overline{\mathcal{S}}_{0, b}$, by (4.6), $z(p) \in$ $\Sigma_{\pi-\epsilon}$. By Lemma 2.1, $z(p) w(z(p)) \in \Sigma_{\pi-\epsilon}$. By (2.1), for small $\epsilon>0$

$$
\left\|\left(z I+A_{h}\right)^{-1}\right\| \leq c /|\Im(z)| \leq c /|z \sin (\pi-\epsilon)| \leq c /(|z| \epsilon) \quad \forall z \in \Sigma_{\pi-\epsilon}^{\prime} .
$$

For any $p \in \overline{\mathcal{S}}_{0, b}, \Re(z(p))=\lambda(1-\sin (\psi+\eta) \cosh (\xi))$. By Lemma 2.1,

$$
\begin{aligned}
\|\widehat{g}(p, t)\| & \leq c e^{\Re(z(p)) t}\left|z^{\prime}(p) w(z(p))\right|\left\|(z(p) w(z(p))+A)^{-1}\right\|\left\|v_{h}\right\| \\
& \leq c \epsilon^{-1} e^{\lambda(1-\sin (\psi+\eta) \cosh (\xi)) t}\left|z^{\prime}(p)\left\|\left.z(p)\right|^{-1}\right\| v_{h} \| .\right.
\end{aligned}
$$

This and (4.6) yield (4.7). The case $p \in \overline{\mathcal{S}}_{a, 0}$ is direct: (4.6) and Lemma 2.1 imply $z(p) w(z(p)) \in \Sigma_{\theta}$. Then (4.7) follows from (4.5) and (2.1).

Now we can give an error estimate on the approximation $U_{N, h}$.

LEMma 4.4. Let $u_{h}(t)$ and $U_{N, h}(t)$ be defined in (4.2) and (4.4), respectively, and the contour be parametrically represented by (4.1). Then with the choice $k=c_{0} / N$ and $\lambda=c_{1} N / t$, there holds

$$
\left\|u_{h}(t)-U_{N, h}(t)\right\| \leq c e^{-c^{\prime} N}\|v\| \text {. }
$$

P r o o f. Let $u_{h}-U_{N, h}=\left(u_{h}-U_{h}\right)+\left(U_{h}-U_{N, h}\right)=: E_{q}+E_{t}$. Let $a=\pi / 2-\psi-\epsilon$ and $b=\psi-\epsilon$. For $p=\xi+\mathrm{i} a, z w(z)$ lies in $\Sigma_{\theta}$ for some 
$\theta \in(\pi / 2, \pi)$. Since $\cosh \xi \geq 1+\xi^{2} / 2$ and $1-\sin (\pi / 2-\epsilon) \leq \epsilon$ for small $\epsilon>0$, the choice $\lambda=c_{1} N / t$ and Lemma 4.3 yield

$$
\begin{aligned}
\left\|\int_{-\infty}^{\infty}|\widehat{g}(\xi+\mathrm{i} a)| d \xi\right\| & \leq c \epsilon^{-1} \int_{0}^{\infty} e^{c_{1} N(1-\sin (\pi / 2-\epsilon) \cosh (\xi))} d \xi\left\|v_{h}\right\| \\
& \leq c \epsilon^{-1} e^{c_{1} N \epsilon} \int_{0}^{\infty} e^{-c_{1} N \sin (\pi / 2-\epsilon) \xi^{2} / 2} d \xi\left\|v_{h}\right\| \\
& \leq c \epsilon^{-1} N^{-1 / 2} e^{c_{1} N \epsilon}\left\|v_{h}\right\| .
\end{aligned}
$$

By Lemma 4.1, for $k=c_{0} / N$ we have

$$
\left\|E_{q}^{+}\right\| \leq c \epsilon^{-1} N^{-\frac{1}{2}} e^{-\left(2 \pi\left(\frac{\pi}{2}-\psi-\epsilon\right) / c_{0}-\epsilon c_{1}\right) N}\left\|v_{h}\right\| .
$$

Next we bound the error due to the lower half. For the choice $p=\xi-\mathrm{i} b$, $\lambda=c_{1} N / t$ and by the inequality $\cosh \xi \geq 1+\xi^{2} / 2$, we deduce

$$
\begin{aligned}
\left\|\int_{-\infty}^{\infty}|\widehat{g}(\xi-\mathrm{i} b)| d \xi\right\| & \leq c \epsilon^{-1} \int_{0}^{\infty} e^{c_{1} N(1-\sin (\epsilon) \cosh (\xi))} d \xi\left\|v_{h}\right\| \\
& \leq c \epsilon^{-1} e^{c_{1} N(1-\sin (\epsilon))} \int_{0}^{\infty} e^{-c_{1} N \sin (\epsilon) \xi^{2} / 2} d \xi\left\|v_{h}\right\| \\
& \leq c \epsilon^{-3 / 2} N^{-1 / 2} e^{c_{1} N(1-\epsilon)}\left\|v_{h}\right\| .
\end{aligned}
$$

Then for the choice $k=c_{0} / N$, Lemma 4.1 yields the following estimate $\left\|E_{q}^{-}\right\| \leq c \epsilon^{-3 / 2} N^{-1 / 2} e^{-\left(2 \pi(\psi-\epsilon) / c_{0}-c_{1}(1-\epsilon)\right) N}\left\|v_{h}\right\|$. Further, since $\cosh (\xi) \geq$ $\cosh \left(c_{0}\right)+\sinh \left(c_{0}\right)\left(\xi-c_{0}\right)$ for $\xi \geq c_{0}$, the error $\left\|E_{t}\right\|$ is bounded by

$$
\begin{aligned}
\left\|E_{t}\right\| & \leq c \epsilon^{-1} \int_{c_{0}}^{\infty} e^{c_{1} N(1-\sin (\psi) \cosh (\xi))} d \xi\left\|v_{h}\right\| \\
& \leq c \epsilon^{-1} e^{c_{1} N\left(1-\sin (\psi) \cosh \left(c_{0}\right)\right)} \int_{c_{0}}^{\infty} e^{-c_{1} N \sin (\psi) \sinh \left(c_{0}\right)\left(\xi-c_{0}\right)} d \xi\left\|v_{h}\right\| \\
& =c /\left(c_{1} \sin (\psi) \sinh \left(c_{0}\right) \epsilon\right) N^{-1} e^{c_{1} N\left[1-\sin (\psi) \cosh \left(c_{0}\right)\right]}\left\|v_{h}\right\| .
\end{aligned}
$$

By disregarding $\epsilon$ terms, balancing the exponentials in $\left\|E_{q}^{+}\right\|,\left\|E_{q}^{-}\right\|$and $\left\|E_{t}\right\|$, we get $2 \pi(\pi / 2-\psi) / c_{0}=2 \pi \psi / c_{0}-c_{1}=-c_{1}\left(1-\sin (\psi) \cosh \left(c_{0}\right)\right)$. Next we express $c_{0}$ and $c_{1}$ using $\psi: c_{0}=\cosh ^{-1}\left(2 \pi \psi /\left(\left(4 \pi \psi-\pi^{2}\right) \sin \psi\right)\right)$ and $c_{1}=\left(4 \pi \psi-\pi^{2}\right) / \cosh ^{-1}\left(2 \pi \psi /\left(\left(4 \pi \psi-\pi^{2}\right) \sin \psi\right)\right)$. Finally we minimize the ratio $B(\psi)=c_{1}-2 \pi \psi / c_{0}$ with respect to $\psi$, which achieves the minimum at $\psi=1.1721$ and hence, $c_{0}=1.0818, c_{1}=4.4920$ and $B(\psi)=-2.32$, which are identical to the values in [32]. Then collecting the balanced asymptotic bound and $\left\|E_{q}^{+}\right\|,\left\|E_{q}^{-}\right\|$and $\left\|E_{t}\right\|$, and choosing $\epsilon=1 / N$ yield

$$
\begin{aligned}
\left\|u_{h}(t)-U_{N, h}(t)\right\| & \leq c \epsilon^{-1}\left(N^{-\frac{1}{2}}+\epsilon^{-\frac{1}{2}} N^{-\frac{1}{2}}+N^{-1}\right) e^{-\left[2.32-\left(\frac{2 \pi}{c_{0}}+c_{1}\right) \epsilon\right] N}\left\|v_{h}\right\| \\
& \leq c e^{\left(-2.32+\frac{\ln N}{N}\right) N}\left\|v_{h}\right\| .
\end{aligned}
$$


Since $x^{-1} \ln x \leq 1 / e$ for $x \geq 1$, the $L^{2}$-stability of $P_{h}$ yields (4.8).

Last, we give error estimates for the fully discrete scheme (4.4). It follows from Theorems 3.1 and 3.2, and Lemma 4.4 .

TheOrem 4.1. Let $u(t)$ be the solution of problem (1.1), and $U_{N, h}(t)$ be the approximation (4.4), with the parameters chosen as in Lemma 4.4 . Then with $\ell_{1}(t)=(\ln 2 T / t)^{-1}$, the following statements hold.

(a) If $A v \in L^{2}(\Omega)$ and $v_{h}=R_{h} v$, then

$$
\left\|u(t)-U_{N, h}(t)\right\| \leq c\left(e^{-c^{\prime} N}+h^{2}\right)\|A v\| .
$$

(b) If $v \in L^{2}(\Omega)$ and $v_{h}=P_{h} v$, then

$$
\left\|u(t)-U_{N, h}\right\| \leq c_{T}\left(e^{-c^{\prime} N}+h^{2} t^{-1} \ell_{1}(t)\right)\|v\| .
$$

\section{Fully Discrete Scheme II: Convolution Quadrature}

Now we develop a second fully discrete scheme based on convolution quadrature. We divide the interval $[0, T]$ into a uniform grid with a time step size $\tau=T / N, N \in \mathbb{N}$, with $t_{n}=n \tau, n=0, \ldots, N$. Following [19, 5], we consider the convolution quadrature generated by the backward Euler method. The weights $\left\{b_{j}\right\}_{j=0}^{\infty}$ are given by [19] $\widetilde{\omega}(\xi)=\sum_{j=0}^{\infty} b_{j} \xi^{j}=$ $\left(\frac{1-\xi}{\tau}\right) w\left(\frac{1-\xi}{\tau}\right)$. Then the convolution quadrature approximation is given by: for $n=1,2, \ldots, N: Q_{n}(\varphi)=\sum_{j=0}^{n} b_{n-j} \varphi\left(t_{j}\right)$. Then we get a fully discrete scheme for (1.1): with $U_{h}^{0}=v_{h}$, for $n=1,2, \ldots, N$

$$
Q_{n}\left(U_{h}-v_{h}\right)+A_{h} U_{h}^{n}=0 .
$$

We denote the generating function $\widetilde{\beta}$ of $\left\{\beta_{j}\right\}_{j=0}^{\infty}$ by $\widetilde{\beta}(\xi)=\sum_{j=0}^{\infty} \beta_{j} \xi^{j}$.

Now we analyze (5.1), following [20]. First we split the error into

$$
e^{n}=u\left(t_{n}\right)-U_{h}^{n}=\left(u\left(t_{n}\right)-u_{h}\left(t_{n}\right)\right)+\left(u_{h}\left(t_{n}\right)-U_{h}^{n}\right) .
$$

By Theorems 3.1 and 3.2 , it suffices to bound $\left\|u_{h}\left(t_{n}\right)-U_{h}^{n}\right\|$. We write $u_{h}\left(t_{n}\right)-U_{h}^{n}=y_{h}(t)-Y_{h}^{n}$, where $y_{h}(t)=u_{h}(t)-v_{h}$ and $Y_{h}^{n}=U_{h}^{n}-v_{h}$.

First, we derive representations of $y_{h}$ and $Y_{h}$.

Lemma 5.1. Let

$$
K(z)=-z^{-1}\left(z w(z) I+A_{h}\right)^{-1} A_{h} \text { and } \chi(z)=\left(1-e^{-z \tau}\right) / \tau .
$$

Then $y_{h}$ and $Y_{h}^{n}$ can be represented by

$$
y_{h}(t)=\frac{1}{2 \pi \mathrm{i}} \int_{\Gamma_{\theta, \delta}} e^{z t} K(z) v_{h} d z \quad \text { and } \quad Y_{h}^{n}=\frac{1}{2 \pi \mathrm{i}} \int_{\Gamma_{\tau}} e^{z t_{n-1}} K(\chi(z)) v_{h} d z,
$$

respectively, with the contour $\Gamma_{\tau}=\left\{z \in \Gamma_{\theta, \delta}:|\Im(z)| \leq \pi / \tau\right\}$. 
P r o o f. By definition, $y_{h}$ satisfies $\mathrm{D}_{t}^{[\mu]} y_{h}+A_{h} y_{h}=-A_{h} v_{h}$, with $y_{h}(0)=0$. The Laplace transform gives $z w(z) \widehat{y}_{h}(z)+A_{h} \widehat{y}_{h}(z)=-z^{-1} A_{h} v_{h}$. Hence, $\widehat{y}_{h}(z)=K(z) v_{h}$, with $K(z)=-z^{-1}\left(z w(z) I+A_{h}\right)^{-1} A_{h}$, and thus follows the representation of $y_{h}(t)$. Likewise, $Y_{h}^{n}$ satisfies

$$
Q_{n}\left(Y_{h}\right)+A Y_{h}^{n}=-A_{h} v_{h} \text { with } Y_{h}^{0}=0 .
$$

Multiplying both sides by $\xi^{n}$ and summing in $n$ from 1 to $\infty$ yield

$$
\sum_{n=1}^{\infty} Q_{n}\left(Y_{h}\right) \xi^{n}+A_{h} \widetilde{Y}_{h}(\xi)=-\xi /(1-\xi) A_{h} v_{h}
$$

Using the condition $Y_{h}^{0}=0$, we have

$$
\sum_{n=1}^{\infty} Q_{n}\left(Y_{h}\right) \xi^{n}=\sum_{n=0}^{\infty} \sum_{j=0}^{n}\left(b_{n-j} \xi^{n-j}\right)\left(Y_{h}^{j} \xi^{j}\right)=((1-\xi) / \tau) w((1-\xi) / \tau) \widetilde{Y}_{h}(\xi) .
$$

Thus, simple calculation shows $\tilde{Y}_{h}(\xi)=(\xi / \tau) K((1-\xi) / \tau) v_{h}$, and it is analytic at $\xi=0$. Then Cauchy theorem implies that for small $\varrho$

$$
Y_{h}^{n}=\frac{1}{2 \tau \pi \mathrm{i}} \int_{|\xi|=\varrho} \xi^{-n} K((1-\xi) / \tau) v_{h} d \xi .
$$

Now, by changing variable $\xi=e^{-z \tau}$, we obtain

$$
Y_{h}^{n}=\frac{1}{2 \pi \mathrm{i}} \int_{\Gamma_{0}} e^{z t_{n-1}} K\left(\left(1-e^{-z \tau}\right) / \tau\right) v_{h} d z,
$$

where the contour $\Gamma_{0}=\{z=-\ln (\varrho) / \tau+\mathrm{i} y:|y| \leq \pi / \tau\}$ is oriented counterclockwise. The desired representation follows by deforming the contour $\Gamma_{0}$ to $\Gamma_{\tau}=\left\{z \in \Gamma_{\theta, \delta}:|\Im(z)| \leq \pi / \tau\right\}$.

By Lemma 5.1, we have $y_{h}\left(t_{n}\right)-Y_{h}^{n}=I+I I$, where

$$
\begin{aligned}
& I=\frac{1}{2 \pi \mathrm{i}} \int_{\Gamma_{\theta, \delta} \backslash \Gamma_{\tau}} e^{z t_{n}} K(z) v_{h} d z, \\
& I I=\frac{1}{2 \pi \mathrm{i}} \int_{\Gamma_{\tau}} e^{z t_{n}}\left(K(z)-e^{-z \tau} K(\chi(z))\right) v_{h} d z .
\end{aligned}
$$

Since $\left|e^{-z \tau}\right|$ is uniformly bounded on $\Gamma_{\tau}$, we have

$$
\begin{aligned}
\left\|K(z)-e^{-z \tau} K(\chi(z))\right\| & \leq\left|e^{-z \tau}\right|\|K(z)-K(\chi(z))\|+\left|1-e^{-z \tau}\right|\|K(z)\| \\
& \leq c\|K(z)-K(\chi(z))\|+c \tau|z|\|K(z)\| \\
& \leq c\|K(z)-K(\chi(z))\|+c \tau,
\end{aligned}
$$

where the last line, using (2.1), follows from the inequality

$$
\|K(z)\|=|z|^{-1}\left\|-I+z w(z)\left(z w(z)+A_{h}\right)^{-1}\right\| \leq c|z|^{-1} .
$$

Hence, it remains to bound the term $\|K(z)-K(\chi(z))\|$. First we recall a bound on $\chi(z)=\tau^{-1}\left(1-e^{-z \tau}\right)$ [13, Lemma 3.1]. 
Lemma 5.2. Let $\chi(z)=\tau^{-1}\left(1-e^{-z \tau}\right)$. Then for all $z \in \Gamma_{\tau}$, there hold

$$
|\chi(z)-z| \leq c|z|^{2} \tau \quad \text { and } \quad c_{1}|z| \leq|\chi(z)| \leq c_{2}|z| \text {, }
$$

and $\chi(z)$ lies in a sector $\Sigma_{\theta^{\prime}}$ for some $\theta^{\prime} \in(\pi / 2, \pi)$.

Next we give one estimate on the approximation $\chi(z) w(\chi(z))$ to $z w(z)$.

Lemma 5.3. For $z \in \Gamma_{\tau}$, there holds

$$
|\chi(z) w(\chi(z))-z w(z)| \leq c \tau|z|^{2} w(|z|) \text {. }
$$

P r o o f. By the intermediate value theorem, for $z \in \Gamma_{\tau}$, we have

$$
\left|\chi(z)^{\alpha}-z^{\alpha}\right|=\alpha\left|\int_{z}^{\chi(z)} s^{\alpha-1} d s\right| \leq \alpha|\chi(z)-z| \max _{\eta \in[0,1]}\left|z_{\eta}\right|^{\alpha-1},
$$

where $z_{\eta}=\eta \chi(z)+(1-\eta) z$ with $\eta \in[0,1]$. Next we claim $\left|z_{\eta}\right|^{-1} \leq c|z|^{-1}$ for $\eta \in[0,1]$. We split $\Gamma_{\tau}$ into $\Gamma_{\tau}=\Gamma_{\tau}^{+} \cup \Gamma_{\tau}^{c} \cup \Gamma_{\tau}^{-}$, with $\Gamma_{\tau}^{ \pm}$being the rays in the upper and lower half plane, and $\Gamma_{\tau}^{c}$ is the circular arc. For $z \in \Gamma_{\tau}^{c}$, Taylor expansion gives $z_{\eta}=z\left(1+\eta \sum_{j=1}^{\infty}(-1)^{j} \frac{z^{j} \tau^{j}}{(j+1) !}\right)$. Since $|z \tau| \leq 1$ for $z \in \Gamma_{\tau}^{c},\left|z_{\eta}\right|^{-1} \leq c|z|^{-1}$ for $z \in \Gamma_{\tau}^{c}$. It remains to consider $z \in \Gamma_{\tau}^{+}$. For $z=r e^{\mathrm{i}(\pi-\theta)}$ with $r \tau \in(\delta, \pi / \sin \theta)$ we have $\chi(z)=$ $\frac{1}{\tau}\left(1-e^{r \tau \cos \theta} e^{-\mathrm{i} r \tau \sin \theta}\right)$, and since $r \tau \sin \theta \leq \pi, \Im(\chi(z)) \geq 0$. Then Lemma 5.2 yields $\left|z_{\eta}\right|>\min (|z|,|\chi(z)|) \cos \frac{\theta}{2} \geq c|z|$. This shows the desired claim. Hence, by Lemma 5.2, for $z \in \Gamma_{\tau}$ there holds $\left|\int_{0}^{1}\left(\chi(z)^{\alpha}-z^{\alpha}\right) \mu(\alpha) d \alpha\right| \leq$ $\int_{0}^{1}\left|\chi(z)^{\alpha}-z^{\alpha}\right| \mu(\alpha) d \alpha \leq c \tau|z| \int_{0}^{1}|z|^{\alpha} \mu(\alpha) d \alpha=c \tau|z|^{2} w(|z|)$.

Next we give an error estimate on the approximation $K(\chi(z))$ to $K(z)$.

Lemma 5.4. For $z \in \Gamma_{\tau}$, there holds $\|K(z)-K(\chi(z))\| \leq c \tau$.

P r o o f. Let $B(z)=z K(z)$. Simple computation shows

$$
\begin{aligned}
B(z)- & B(\chi(z))=z w(z)\left(\left(z w(z) I+A_{h}\right)^{-1}-\left(\chi(z) w(\chi(z)) I+A_{h}\right)^{-1}\right) \\
+ & (z w(z)-\chi(z) w(\chi(z)))\left(\chi(z) w(\chi(z)) I+A_{h}\right)^{-1}:=I+I I .
\end{aligned}
$$

First, by Lemmas 2.3 and 5.2 , there holds $|\chi(z) w(\chi(z))| \geq c|\chi(z)| w(|\chi(z)|)$ $\geq c|z| w(|z|)$. Further, by Lemma 2.1, (2.1) and Lemma 2.3, we have

$$
\left\|\left(z w(z) I+A_{h}\right)^{-1}\right\| \leq c|z w(z)|^{-1} \leq c(|z| w(|z|))^{-1} .
$$

Likewise, in view of Lemmas 5.2 and 2.1 and (2.1), we have

$$
\left\|\left(\chi(z) w(\chi(z)) I+A_{h}\right)^{-1}\right\| \leq c|\chi(z) w(\chi(z))|^{-1} \leq c(|z| w(|z|))^{-1} .
$$

Now, by the identity 


$$
\begin{aligned}
& \left(z w(z) I+A_{h}\right)^{-1}-\left(\chi(z) w(\chi(z)) I+A_{h}\right)^{-1} \\
= & (z w(z)-\chi(z) w(\chi(z)))\left(z w(z) I+A_{h}\right)^{-1}\left(\chi(z) w(\chi(z)) I+A_{h}\right)^{-1},
\end{aligned}
$$

Lemma 2.3, (5.4) and (5.5), the first term $I$ can be bounded by

$$
\|I\| \leq c \tau|z|^{3} w(|z|)^{2}\left\|\left(z w(z) I+A_{h}\right)^{-1}\right\|\left\|\left(\chi(z) w(\chi(z)) I+A_{h}\right)^{-1}\right\| \leq c \tau|z| .
$$

Likewise, by Lemma 5.3 and (5.5)

$$
\begin{aligned}
\|I I\| & \leq|z w(z)-\chi(z) w(\chi(z))|\left\|\left(\chi(z) w(\chi(z)) I+A_{h}\right)^{-1}\right\| \\
& \leq c \tau|z|^{2} w(|z|)|z w(|z|)|^{-1} \leq c \tau|z| .
\end{aligned}
$$

Hence, $\|B(z)-B(\chi(z))\| \leq c \tau|z|$. Last, by Lemma 5.2 and $\|B(z)\| \leq c$,

$$
\begin{aligned}
\|K(z)-K(\chi(z))\| & \leq\left|z^{-1}-\chi(z)^{-1}\right|\|B(z)\|+|z|^{-1}\|B(z)-B(\chi(z))\| \\
& \leq c|z-\chi(z)||z|^{-2}+c \tau \leq c \tau .
\end{aligned}
$$

Now we can state an error estimate for nonsmooth data $v \in L^{2}(\Omega)$.

TheOREM 5.1. Let $u_{h}$ and $U_{h}^{n}$ be the solutions of problems (3.1) and (5.1) with $v \in L^{2}(\Omega), v_{h}=P_{h} v$ and $f \equiv 0$, respectively. Then there holds

$$
\left\|u_{h}\left(t_{n}\right)-U_{h}^{n}\right\| \leq c \tau t_{n}^{-1}\|v\| .
$$

$\mathrm{P}$ r o o f. It suffices to bound the terms $I$ and $I I$ in (5.3). By choosing $\delta=t_{n}^{-1}$ in $\Gamma_{\delta, \theta}$ and (2.1), we bound $I$ by

$$
\|I\| \leq c \int_{\frac{\pi}{\tau \sin \theta}}^{\infty} e^{r t_{n} \cos \theta} r^{-1} d r\left\|v_{h}\right\| \leq c \tau\left\|v_{h}\right\| \int_{0}^{\infty} e^{r t_{n} \cos \theta} d r \leq c \tau t_{n}^{-1}\left\|v_{h}\right\| .
$$

Using Lemma 5.4, we deduce

$$
\|I I\| \leq c \tau\left\|v_{h}\right\|\left(\int_{t_{n}^{-1}}^{\frac{\pi}{\tau \sin \theta}} e^{r t_{n} \cos \theta} d r+\int_{-\theta}^{\theta} e^{\cos \psi} t_{n}^{-1} d \psi\right) \leq c t_{n}^{-1} \tau\left\|v_{h}\right\| .
$$

Hence, $\left\|y_{h}\left(t_{n}\right)-Y_{h}^{n}\right\| \leq c \tau t_{n}^{-1}\left\|v_{h}\right\|$, and the estimate follows from $U_{h}^{n}-$ $u_{h}\left(t_{n}\right)=Y_{h}^{n}-y_{h}\left(t_{n}\right)$ and the $L^{2}$ stability of $P_{h}$.

Next we turn to smooth data $A v \in L^{2}(\Omega)$.

Lemma 5.5. Let $K^{s}(z)=-z^{-1}\left(z w(z) I+A_{h}\right)^{-1}$. Then for any $z \in \Gamma_{\tau}$,

$$
\left\|K^{s}(z)-K^{s}(\chi(z))\right\| \leq c \tau(|z|-1)^{-1} \ln |z| .
$$


P r o o f. Let $B^{s}(z)=-\left(z w(z) I+A_{h}\right)^{-1}$. Then by the identity $B^{s}(z)-$ $B^{s}(\chi(z))=(\chi(z) w(\chi(z))-z w(z))\left(z w(z) I+A_{h}\right)^{-1}\left(\chi(z) w(\chi(z)) I+A_{h}\right)^{-1}$, Lemma 5.3, and (5.4) and (5.5), we deduce $\left\|B^{s}(z)-B^{s}(\chi(z))\right\| \leq c \tau|w(z)|^{-1}$. Appealing to (5.4) again yields $\left\|B^{s}(z)\right\| \leq c|z w(z)|^{-1}$, and thus

$$
\begin{gathered}
\left\|K^{s}(z)-K^{s}(\chi(z))\right\| \leq\left|z^{-1}-\chi(z)^{-1}\right||| B^{s}(z)\left\|+|\chi(z)|^{-1}\right\| B^{s}(z)-B^{s}(\chi(z)) \| \\
\leq c|z-\chi(z)||z|^{-3}|w(z)|^{-1}+c \tau|z w(z)|^{-1} \leq c \tau|z w(z)|^{-1} .
\end{gathered}
$$

Then the desired result follows from Lemma 2.3 .

Theorem 5.2. Let $u_{h}$ and $U_{h}^{n}$ be the solutions of problems (3.1) and (5.1) with $A v \in L^{2}(\Omega)$ and $v_{h}=R_{h} v$, respectively. Then with $\ell_{2}(t)=$ $\ln \left(\max \left(t^{-1}, 2\right)\right)$,

$$
\left\|u_{h}\left(t_{n}\right)-U_{h}^{n}\right\| \leq c \tau \ell_{2}(t)\|A v\|
$$

P r o o f. Let $K^{s}(z)=-z^{-1}\left(z w(z) I+A_{h}\right)^{-1}$. Then

$$
\begin{aligned}
y_{h}\left(t_{n}\right)- & Y_{h}^{n}=\frac{1}{2 \pi \mathrm{i}} \int_{\Gamma_{\theta, \delta} \backslash \Gamma_{\tau}} e^{z t_{n}} K^{s}(z) A_{h} v_{h} d z \\
& +\frac{1}{2 \pi \mathrm{i}} \int_{\Gamma_{\tau}} e^{z t_{n}}\left(K^{s}(z)-e^{-z \tau} K^{s}(\chi(z))\right) A_{h} v_{h} d z:=I+I I .
\end{aligned}
$$

By Lemma 5.5, $\left\|K^{s}(z)-e^{-z \tau} K^{s}(\chi(z))\right\| \leq c \tau \ln |z| /(|z|-1)$ for $z \in \Gamma_{\tau}$. With $\delta=t_{n}^{-1}$, the monotonicity of $f(x)=(1-x)^{-1} \ln x$ yields

$$
\begin{aligned}
\|I I\| & \leq c \tau\left\|A_{h} v_{h}\right\|\left(\int_{t_{n}^{-1}}^{\frac{\pi}{\tau \sin \theta}} e^{r t_{n} \cos \theta} \frac{\ln r}{r-1} d r+\int_{-\theta}^{\theta} e^{\cos \psi} \frac{\ln \left(t_{n}^{-1}\right)}{1-t_{n}} d \psi\right) \\
& \leq c\left(1-t_{n}\right)^{-1} \ln \left(t_{n}^{-1}\right) \tau\left\|A_{h} v_{h}\right\| .
\end{aligned}
$$

By (2.1), for $z \in \Gamma_{\theta, \delta},\left\|K^{s}(z)\right\| \leq c|z|^{-1}|z w(z)|^{-1}$. Now Lemma 2.3 yields

$$
\begin{aligned}
\|I\| & \leq c\left\|A_{h} v_{h}\right\| \int_{\frac{\pi}{\tau \sin \theta}}^{\infty} e^{r t_{n} \cos \theta} r^{-2}|w(r)|^{-1} d r \\
& \leq c \tau\left\|A_{h} v_{h}\right\| \int_{t_{n}^{-1}}^{\infty} e^{r t_{n} \cos \theta} \frac{\ln r}{r-1} d r \leq c \frac{\ln \left(t_{n}^{-1}\right)}{1-t_{n}} \tau\left\|A_{h} v_{h}\right\| .
\end{aligned}
$$

Now the identity $A_{h} R_{h}=P_{h} A$ completes the proof.

The next theorem gives error estimates for the scheme (5.1), which follow from Theorems 3.1, 3.2, 5.1 and 5.2.

TheOREM 5.3. Let $u$ and $U_{h}^{n}$ be the solutions of problems (1.1) and (5.1) with $U_{h}^{0}=v_{h}$, respectively. Then the following statements hold. 
(a) If $A v \in L^{2}(\Omega)$ and $v_{h}=R_{h} v$, then with $\ell_{1}(t)=\ln (2 T / t)^{-1}$

$$
\left\|u\left(t_{n}\right)-U_{h}^{n}\right\| \leq c\left(\tau \ell_{2}\left(t_{n}\right)+h^{2}\right)\|A v\| .
$$

(b) If $v \in L^{2}(\Omega)$ and $v_{h}=P_{h} v$, then with $\ell_{2}(t)=\ln \left(\max \left(t^{-1}, 2\right)\right)$

$$
\left\|u\left(t_{n}\right)-U_{h}^{n}\right\| \leq c_{T}\left(\tau+h^{2} \ell_{1}\left(t_{n}\right)\right) t_{n}^{-1}\|v\| .
$$

\section{Numerical Experiments and Discussions}

Now we present numerical results to verify the convergence theory. Throughout, let the domain $\Omega=(0,1)$ and consider

(a) $v(x)=\sin (2 \pi x) \in H^{2}(\Omega) \cap H_{0}^{1}(\Omega)$;

(b) $v=\chi_{(0,1 / 2)} \in H^{1 / 2-\epsilon}(\Omega)$ with $\epsilon \in(0,1 / 2)$, and $\chi_{S}$ the characteristic function of a set $S$;

(c) $v(x)=x^{-1 / 4} \in H^{1 / 4-\epsilon}(\Omega)$ with $\epsilon \in(0,1 / 4)$.

We measure the temporal error by the $L^{2}$ errors $\left\|u\left(t_{n}\right)-U_{N, h}\left(t_{n}\right)\right\| /\|v\|$ or $\left\|u\left(t_{n}\right)-U_{h}^{n}\right\| /\|v\|$, and the spatial error by $\left\|u(t)-u_{h}(t)\right\| /\|v\|$ and $\| \nabla(u(t)-$ $\left.u_{h}(t)\right)\|/\| v \|$. Throughout we divide the domain $\Omega$ into $M$ equally spaced subintervals with a mesh size $h=1 / M$. Since the exact solution $u(t)$ is not unavailable, we compute a reference solution using a finer mesh.

6.1. Numerical results for the semidiscrete scheme. First we examine the convergence behavior of (1.3). Here we fix $N=10$ in the Laplace transform approach such that the error due to time discretization is negligible. The numerical results are given in Table 1 . In the table, rate denotes the empirical rates when the mesh size $h$ halves, and the numbers in the bracket denote the theoretical rates. For all cases, the $L^{2}$ and $H^{1}$ norms of the error exhibit $O\left(h^{2}\right)$ and $O(h)$ convergence, respectively, agreeing with Theorems 3.1 and 3.2. The scheme is robust since the convergence rates hold for both smooth and nonsmooth data. The error increases as $t \rightarrow 0$, due to the weak solution singularity around $t \rightarrow 0$, cf. Theorem 2.1 ,

6.2. Numerical results for the fully discrete scheme I. Next we illustrate the convergence of the scheme (4.4). To make the spatial discretization error negligible, we fix $h$ at $h=10^{-5}$. The numerical results are summarized in Tables 2 and 3 for $\mu(\alpha)=(\alpha-1 / 2)^{2}$ and $\mu(\alpha)=\chi_{[1 / 2,1]}(\alpha)$, respectively. The notation $r$ denotes the exponent in the estimate $\| u_{N, h}^{n}-$ $u\left(t_{n}\right) \| \leq C e^{-r N}$. The results indicate an exponential convergence with respect to $N$, with a rate $e^{-2.15 N}$ and $e^{-2.14 N}$ for $\mu(\alpha)=(\alpha-1 / 2)^{2}$ and $\mu(\alpha)=\chi_{[1 / 2,1]}(\alpha)$, respectively, which agree with Theorem 4.1. Note that even though $\mu(\alpha)=\chi_{[1 / 2,1]}(\alpha)$ does not satisfy the condition $\mu(0) \mu(1)>0$, the empirical rates still agree well with the theoretical one, which calls for further study. The convergence rate is independent of $t$, and thus the 
ERROR ESTIMATES FOR APPROXIMATIONS OF ...

\begin{tabular}{|c|c|c|c|c|c|c|c|c|c|}
\hline 促 & & $M$ & 10 & 20 & 40 & 80 & 160 & 320 & \\
\hline & 1 & \begin{tabular}{|l|}
$L^{2}$ \\
\end{tabular} & $2.79 \mathrm{e}-5$ & $7.02 \mathrm{e}-6$ & $1.76 \mathrm{e}-6$ & $4.39 \mathrm{e}-7$ & $1.09 \mathrm{e}-7$ & $2.70 \mathrm{e}-8$ & 2.0 \\
\hline & & $H^{1}$ & $84 \mathrm{e}-4$ & $44 \epsilon$ & 2.22 & 11 & $5.23 \mathrm{e}-$ & $2.36 \mathrm{e}-5$ & \\
\hline & & $L^{2}$ & 3 & 3 & $4.03 \mathrm{e}-4$ & $1.01 \mathrm{e}-4$ & $2.51 \mathrm{e}-5$ & $6.21 \mathrm{e}-6$ & $2.00(2.0$ \\
\hline & & $H^{1}$ & $41 \mathrm{e}-1$ & $7.04 \mathrm{e}-2$ & $3.53 \mathrm{e}-2$ & $1.76 \mathrm{e}-2$ & $3.75 \mathrm{e}-3$ & $1.65 \mathrm{e}-3$ & 1.01 \\
\hline & & \begin{tabular}{|l|}
$L^{2}$ \\
\end{tabular} & & & & & 1. & $3.87 \mathrm{e}-8$ & $2.00(2$. \\
\hline & & $H^{1}$ & $26 \mathrm{e}-3$ & $.29 \mathrm{e}-4$ & & $1.55 \mathrm{e}-4$ & 7.63e-5 & $3.68 \mathrm{e}-5$ & 1.01 \\
\hline & & \begin{tabular}{|l|}
$L^{2}$ \\
\end{tabular} & $4 \mathrm{e}-3$ & $59 \mathrm{e}-3$ & $3.96 \mathrm{e}-4$ & $9.92 \mathrm{e}-5$ & $2.48 \mathrm{e}-5$ & $6.18 \mathrm{e}-6$ & $2.00(2.0$ \\
\hline & & $H^{1}$ & $73 \mathrm{e}-1$ & & & & $1.04 \mathrm{e}-2$ & & 1.02 \\
\hline & & \begin{tabular}{|l|}
$L^{2}$ \\
\end{tabular} & $82 \mathrm{e}-5$ & & $2.44 \mathrm{e}-6$ & $6.12 \mathrm{e}-7$ & $1.53 \mathrm{e}-7$ & $3.79 \mathrm{e}-8$ & $2.00(2.00$ \\
\hline & & $H^{1}$ & & & & & & & $1.05(1.00$ \\
\hline & $10^{-3}$ & \begin{tabular}{|l|}
$L^{2}$ \\
\end{tabular} & & & $\mathrm{e}-4$ & $5.49 \mathrm{e}-5$ & $1.37 \mathrm{e}-5$ & $3.36 \mathrm{e}-6$ & $2.00(2.00$ \\
\hline & & & & & & & & & 1.01 \\
\hline
\end{tabular}

TABLE 1. Numerical results for the semidiscrete scheme (1.3) with $\mu(\alpha)=(\alpha-1 / 2)^{2}$.

\begin{tabular}{|c|c|cccccc|c|}
\hline case & $t \backslash N$ & 3 & 5 & 7 & 9 & 11 & 13 & $r$ \\
\hline \multirow{3}{*}{ (a) } & 1 & $1.33 \mathrm{e}-6$ & $1.49 \mathrm{e}-8$ & $1.26 \mathrm{e}-10$ & $2.20 \mathrm{e}-12$ & $3.54 \mathrm{e}-14$ & $8.24 \mathrm{e}-17$ & 2.35 \\
& $10^{-2}$ & $4.78 \mathrm{e}-6$ & $7.36 \mathrm{e}-7$ & $2.77 \mathrm{e}-9$ & $5.45 \mathrm{e}-11$ & $4.88 \mathrm{e}-13$ & $2.23 \mathrm{e}-14$ & 1.92 \\
& $10^{-3}$ & $8.30 \mathrm{e}-5$ & $8.78 \mathrm{e}-7$ & $3.81 \mathrm{e}-9$ & $7.55 \mathrm{e}-11$ & $6.43 \mathrm{e}-13$ & $1.23 \mathrm{e}-14$ & 2.26 \\
\hline \multirow{3}{*}{ (b) } & 1 & $3.34 \mathrm{e}-6$ & $3.56 \mathrm{e}-8$ & $2.85 \mathrm{e}-10$ & $5.76 \mathrm{e}-12$ & $8.68 \mathrm{e}-14$ & $1.25 \mathrm{e}-15$ & 2.17 \\
& $10^{-2}$ & $1.24 \mathrm{e}-5$ & $8.29 \mathrm{e}-7$ & $2.31 \mathrm{e}-9$ & $6.09 \mathrm{e}-11$ & $4.78 \mathrm{e}-13$ & $2.18 \mathrm{e}-14$ & 2.02 \\
& $10^{-3}$ & $6.99 \mathrm{e}-5$ & $1.73 \mathrm{e}-6$ & $1.09 \mathrm{e}-8$ & $5.38 \mathrm{e}-11$ & $1.17 \mathrm{e}-12$ & $1.59 \mathrm{e}-14$ & 2.22 \\
\hline \multirow{3}{*}{ (c) } & 1 & $8.04 \mathrm{e}-6$ & $9.05 \mathrm{e}-8$ & $6.80 \mathrm{e}-10$ & $1.39 \mathrm{e}-11$ & $2.08 \mathrm{e}-13$ & $3.02 \mathrm{e}-15$ & 2.17 \\
& $10^{-2}$ & $3.01 \mathrm{e}-5$ & $1.71 \mathrm{e}-6$ & $3.85 \mathrm{e}-9$ & $1.26 \mathrm{e}-10$ & $9.22 \mathrm{e}-13$ & $4.21 \mathrm{e}-14$ & 2.04 \\
& $10^{-3}$ & $1.16 \mathrm{e}-4$ & $4.09 \mathrm{e}-6$ & $2.65 \mathrm{e}-8$ & $6.65 \mathrm{e}-11$ & $2.75 \mathrm{e}-12$ & $3.49 \mathrm{e}-14$ & 2.19 \\
\hline
\end{tabular}

TABLE 2. The $L^{2}$ errors for (a)-(c) with $h=10^{-5}$ and $\mu(\alpha)=(\alpha-1 / 2)^{2}$, by the Laplace transform method.

scheme is robust. Interestingly, the smoothness of the initial data does not affect much the time discretization errors, even for small time, cf. Table 4.

One salient feature of the fully discrete scheme I is that it allows computing the solution at large time. This allows one to examine the asymptotic behavior of the solution as the time $t \rightarrow \infty$; see Table 5 and Fig. 1) In particular, one clearly observes the logarithmic decay of the solution [18, Theorem 2.1]; see also Fig. 11. This numerically verifies the ultraslow decay asymptotics for distributed order diffusion process. 


\begin{tabular}{|c|c|cccccc|c|}
\hline case & $t \backslash N$ & 3 & 5 & 7 & 9 & 11 & 13 & $r$ \\
\hline \multirow{3}{*}{ (a) } & 1 & $4.54 \mathrm{e}-6$ & $2.30 \mathrm{e}-7$ & $1.63 \mathrm{e}-9$ & $1.69 \mathrm{e}-11$ & $2.36 \mathrm{e}-13$ & $8.46 \mathrm{e}-15$ & 2.02 \\
& $10^{-2}$ & $6.21 \mathrm{e}-5$ & $1.65 \mathrm{e}-6$ & $3.71 \mathrm{e}-9$ & $1.07 \mathrm{e}-10$ & $7.00 \mathrm{e}-13$ & $2.58 \mathrm{e}-14$ & 2.16 \\
& $10^{-3}$ & $8.02 \mathrm{e}-4$ & $3.61 \mathrm{e}-6$ & $1.66 \mathrm{e}-8$ & $4.17 \mathrm{e}-10$ & $3.10 \mathrm{e}-12$ & $6.73 \mathrm{e}-15$ & 2.55 \\
\hline \multirow{3}{*}{ (b) } & 1 & $4.78 \mathrm{e}-6$ & $4.74 \mathrm{e}-7$ & $2.43 \mathrm{e}-9$ & $3.44 \mathrm{e}-11$ & $3.49 \mathrm{e}-13$ & $1.87 \mathrm{e}-14$ & 1.94 \\
& $10^{-2}$ & $1.03 \mathrm{e}-4$ & $1.13 \mathrm{e}-6$ & $3.58 \mathrm{e}-9$ & $8.78 \mathrm{e}-11$ & $5.04 \mathrm{e}-13$ & $1.93 \mathrm{e}-14$ & 2.24 \\
& $10^{-3}$ & $5.12 \mathrm{e}-4$ & $4.79 \mathrm{e}-6$ & $4.95 \mathrm{e}-8$ & $5.23 \mathrm{e}-10$ & $5.15 \mathrm{e}-12$ & $5.58 \mathrm{e}-14$ & 2.29 \\
\hline \multirow{3}{*}{ (c) } & 1 & $4.79 \mathrm{e}-6$ & $5.61 \mathrm{e}-7$ & $2.75 \mathrm{e}-9$ & $4.07 \mathrm{e}-11$ & $3.94 \mathrm{e}-13$ & $2.23 \mathrm{e}-14$ & 1.92 \\
& $10^{-2}$ & $1.18 \mathrm{e}-4$ & $6.08 \mathrm{e}-7$ & $3.37 \mathrm{e}-9$ & $7.22 \mathrm{e}-11$ & $2.84 \mathrm{e}-13$ & $8.94 \mathrm{e}-14$ & 2.10 \\
& $10^{-3}$ & $1.09 \mathrm{e}-4$ & $5.24 \mathrm{e}-6$ & $6.02 \mathrm{e}-8$ & $5.62 \mathrm{e}-10$ & $5.95 \mathrm{e}-12$ & $1.02 \mathrm{e}-13$ & 2.07 \\
\hline
\end{tabular}

TABLE 3. The $L^{2}$ errors for (a)-(c) with $h=10^{-5}$ and $\mu(\alpha)=\chi_{[1 / 2,1]}(\alpha)$, by the Laplace transform method.

\begin{tabular}{|c|cccccc|}
\hline case $\backslash t$ & $10^{-4}$ & $10^{-5}$ & $10^{-6}$ & $10^{-7}$ & $10^{-8}$ & $10^{-9}$ \\
\hline (b) & $7.05 \mathrm{e}-6$ & $9.39 \mathrm{e}-6$ & $1.58 \mathrm{e}-5$ & $1.75 \mathrm{e}-5$ & $1.81 \mathrm{e}-5$ & $1.82 \mathrm{e}-5$ \\
\hline (c) & $6.39 \mathrm{e}-6$ & $1.17 \mathrm{e}-5$ & $1.53 \mathrm{e}-5$ & $1.68 \mathrm{e}-5$ & $1.75 \mathrm{e}-5$ & $1.79 \mathrm{e}-5$ \\
\hline
\end{tabular}

TABLE 4. The $L^{2}$ errors for (b) and (c) with $h=10^{-5}$, $\mu(\alpha)=(\alpha-1 / 2)^{2}$ and $N=5$ at time $t=10^{-k}, k=4, \ldots, 9$, by the Laplace transform method.

\begin{tabular}{|c|ccccccc|c|}
\hline case $\backslash k$ & 6 & 8 & 10 & 12 & 14 & 16 & 18 & rate \\
\hline (a) & $3.33 \mathrm{e}-4$ & $2.70 \mathrm{e}-4$ & $2.26 \mathrm{e}-4$ & $1.95 \mathrm{e}-4$ & $1.71 \mathrm{e}-4$ & $1.52 \mathrm{e}-4$ & $1.37 \mathrm{e}-4$ & $1 / k$ \\
\hline (c) & $1.06 \mathrm{e}-3$ & $8.54 \mathrm{e}-4$ & $7.17 \mathrm{e}-4$ & $6.17 \mathrm{e}-4$ & $5.41 \mathrm{e}-4$ & $4.82 \mathrm{e}-4$ & $4.34 \mathrm{e}-4$ & $1 / k$ \\
\hline
\end{tabular}

TABLE 5. The $L^{2}$ norm of the solution for (a) and (c) with $h=10^{-5}, \mu(\alpha)=(\alpha-1 / 2)^{2}$ and $N=10$ at time $t=10^{k}$, $k=6,8, \cdots, 18$, by the Laplace transform method.

6.3. Numerical results for the fully discrete scheme II. Last we verify the convergence of the fully discrete scheme II, i.e., (5.1). The results are shown in Tables 6 and 7 for $\mu(\alpha)=(\alpha-1 / 2)^{2}$ and $\mu(\alpha)=\chi_{[1 / 2,1]}(\alpha)$, respectively. An $O(\tau)$ convergence is always observed, cf. Theorem 5.3.

To examine more closely its convergence behavior, we consider $t=10^{-k}$, $k=4, \ldots, 9$, and at each time $t$, divide the interval $[0, t]$ into $N=10$ subintervals. The scheme works well for the smooth initial data, however, it works poorly for the singular initial data, cf. Table 8 . This behavior is predicted by Theorem [5.1; the error is dominated by the factor $\tau / t$ for $L^{2}$ initial data. In Fig. 2, we plot the ratio $\left\|U_{h}^{1}-u(\tau)\right\| / \tau$ versus $\ln \tau$ for 


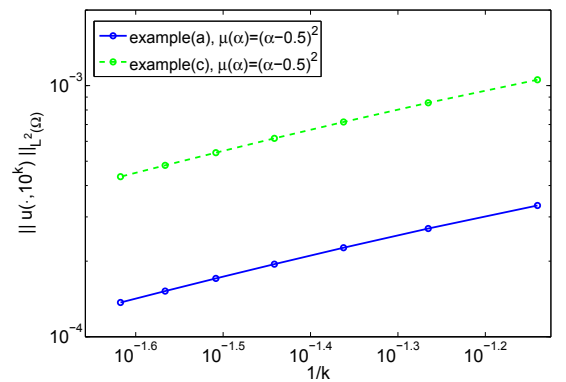

Figure 1. The $L^{2}$ norm of the solution for (a) and (c) at $t=10^{k}, k=6,8, \cdots, 18$, by the Laplace transform method.

\begin{tabular}{|c|c|cccccc|c|}
\hline case & $t \backslash N$ & 10 & 20 & 40 & 80 & 160 & 320 & rate \\
\hline \multirow{3}{*}{ (a) } & 1 & $1.82 \mathrm{e}-5$ & $8.78 \mathrm{e}-6$ & $4.31 \mathrm{e}-6$ & $2.12 \mathrm{e}-6$ & $1.01 \mathrm{e}-6$ & $4.74 \mathrm{e}-7$ & $1.05(1.00)$ \\
& $10^{-2}$ & $8.64 \mathrm{e}-4$ & $3.91 \mathrm{e}-4$ & $1.88 \mathrm{e}-4$ & $9.20 \mathrm{e}-5$ & $4.55 \mathrm{e}-5$ & $2.26 \mathrm{e}-5$ & $1.05(1.00)$ \\
& $10^{-3}$ & $2.17 \mathrm{e}-2$ & $1.10 \mathrm{e}-2$ & $5.51 \mathrm{e}-3$ & $2.76 \mathrm{e}-3$ & $1.38 \mathrm{e}-3$ & $6.92 \mathrm{e}-4$ & $0.99(1.00)$ \\
\hline \multirow{3}{*}{ (b) } & 1 & $4.81 \mathrm{e}-5$ & $2.32 \mathrm{e}-5$ & $1.14 \mathrm{e}-5$ & $5.60 \mathrm{e}-6$ & $2.67 \mathrm{e}-6$ & $1.26 \mathrm{e}-6$ & $1.05(1.00)$ \\
& $10^{-2}$ & $8.11 \mathrm{e}-3$ & $3.87 \mathrm{e}-3$ & $1.88 \mathrm{e}-3$ & $9.29 \mathrm{e}-4$ & $4.61 \mathrm{e}-4$ & $2.30 \mathrm{e}-4$ & $1.03(1.00)$ \\
& $10^{-3}$ & $1.48 \mathrm{e}-2$ & $7.46 \mathrm{e}-3$ & $3.74 \mathrm{e}-3$ & $1.88 \mathrm{e}-3$ & $9.39 \mathrm{e}-4$ & $4.70 \mathrm{e}-4$ & $1.00(1.00)$ \\
\hline \multirow{3}{*}{ (c) } & 1 & $5.81 \mathrm{e}-5$ & $2.81 \mathrm{e}-5$ & $1.38 \mathrm{e}-5$ & $6.76 \mathrm{e}-6$ & $3.23 \mathrm{e}-6$ & $1.52 \mathrm{e}-6$ & $1.05(1.00)$ \\
& $10^{-2}$ & $1.01 \mathrm{e}-2$ & $4.80 \mathrm{e}-3$ & $2.34 \mathrm{e}-3$ & $1.15 \mathrm{e}-3$ & $5.72 \mathrm{e}-4$ & $2.85 \mathrm{e}-4$ & $1.03(1.00)$ \\
& $10^{-3}$ & $7.35 \mathrm{e}-3$ & $3.66 \mathrm{e}-3$ & $1.82 \mathrm{e}-3$ & $9.11 \mathrm{e}-4$ & $4.55 \mathrm{e}-4$ & $2.27 \mathrm{e}-4$ & $1.00(1.00)$ \\
\hline
\end{tabular}

TABLE 6. The $L^{2}$ errors for (a)-(c) with $h=10^{-4}$ and $\mu(\alpha)=(\alpha-1 / 2)^{2}$, by convolution quadrature.

\begin{tabular}{|c|c|cccccc|c|}
\hline case & $t \backslash N$ & 10 & 20 & 40 & 80 & 160 & 320 & rate \\
\hline \multirow{3}{*}{ (a) } & 1 & $2.20 \mathrm{e}-4$ & $1.06 \mathrm{e}-4$ & $5.20 \mathrm{e}-5$ & $2.58 \mathrm{e}-5$ & $1.28 \mathrm{e}-5$ & $6.40 \mathrm{e}-6$ & $1.02(1.00)$ \\
& $10^{-2}$ & $1.76 \mathrm{e}-2$ & $8.81 \mathrm{e}-3$ & $4.40 \mathrm{e}-3$ & $2.20 \mathrm{e}-3$ & $1.10 \mathrm{e}-3$ & $5.49 \mathrm{e}-4$ & $1.00(1.00)$ \\
& $10^{-3}$ & $3.92 \mathrm{e}-3$ & $1.98 \mathrm{e}-3$ & $9.95 \mathrm{e}-4$ & $4.99 \mathrm{e}-4$ & $2.50 \mathrm{e}-4$ & $1.25 \mathrm{e}-4$ & $0.99(1.00)$ \\
\hline & 1 & $6.52 \mathrm{e}-4$ & $3.11 \mathrm{e}-4$ & $1.52 \mathrm{e}-4$ & $7.53 \mathrm{e}-5$ & $3.74 \mathrm{e}-5$ & $1.87 \mathrm{e}-5$ & $1.03(1.00)$ \\
(b) & $10^{-2}$ & $1.25 \mathrm{e}-2$ & $6.26 \mathrm{e}-3$ & $3.13 \mathrm{e}-3$ & $1.56 \mathrm{e}-3$ & $7.82 \mathrm{e}-4$ & $3.91 \mathrm{e}-4$ & $1.00(1.00)$ \\
& $10^{-3}$ & $5.76 \mathrm{e}-3$ & $2.88 \mathrm{e}-3$ & $1.44 \mathrm{e}-3$ & $7.18 \mathrm{e}-4$ & $3.59 \mathrm{e}-4$ & $1.79 \mathrm{e}-4$ & $1.00(1.00)$ \\
\hline \multirow{3}{*}{ (c) } & 1 & $7.92 \mathrm{e}-4$ & $3.78 \mathrm{e}-4$ & $1.85 \mathrm{e}-4$ & $9.14 \mathrm{e}-5$ & $4.54 \mathrm{e}-5$ & $2.27 \mathrm{e}-5$ & $1.03(1.00)$ \\
& $10^{-2}$ & $7.40 \mathrm{e}-3$ & $3.71 \mathrm{e}-3$ & $1.86 \mathrm{e}-3$ & $9.28 \mathrm{e}-3$ & $4.64 \mathrm{e}-4$ & $2.32 \mathrm{e}-4$ & $1.00(1.00)$ \\
& $10^{-3}$ & $6.10 \mathrm{e}-3$ & $3.06 \mathrm{e}-3$ & $1.53 \mathrm{e}-3$ & $7.65 \mathrm{e}-4$ & $3.83 \mathrm{e}-4$ & $1.91 \mathrm{e}-4$ & $1.00(1.00)$ \\
\hline
\end{tabular}

TABLE 7. The $L^{2}$ errors for (a)-(c) with $h=10^{-4}$ and $\mu(\alpha)=\chi_{[1 / 2,1]}(\alpha)$, by convolution quadrature. 
smooth initial data. Theorem 5.2 predicts $\left\|U_{h}^{1}-u(\tau)\right\| \leq c \tau \ln \tau^{-1}$. The $\log$ factor $\ell_{2}(t)$ in Theorem 5.2 is confirmed by Fig. 2, and thus it is sharp.

\begin{tabular}{|c|cccccc|c|}
\hline case $\backslash t$ & $10^{-4}$ & $10^{-5}$ & $10^{-6}$ & $10^{-7}$ & $10^{-8}$ & $10^{-9}$ & rate \\
\hline (a) & $2.42 \mathrm{e}-3$ & $1.03 \mathrm{e}-4$ & $7.87 \mathrm{e}-6$ & $7.59 \mathrm{e}-7$ & $7.58 \mathrm{e}-8$ & $7.44 \mathrm{e}-9$ & $1.01(1.00)$ \\
\hline (c) & $7.44 \mathrm{e}-3$ & $5.67 \mathrm{e}-3$ & $4.30 \mathrm{e}-3$ & $3.27 \mathrm{e}-3$ & $2.49 \mathrm{e}-3$ & $1.88 \mathrm{e}-3$ & $0.12(0.12)$ \\
\hline
\end{tabular}

TABLE 8 . The $L^{2}$ errors with $h=10^{-5}$ and $N=10$, at $t=10^{-k}, k=4, \ldots, 9$, by convolution quadrature.

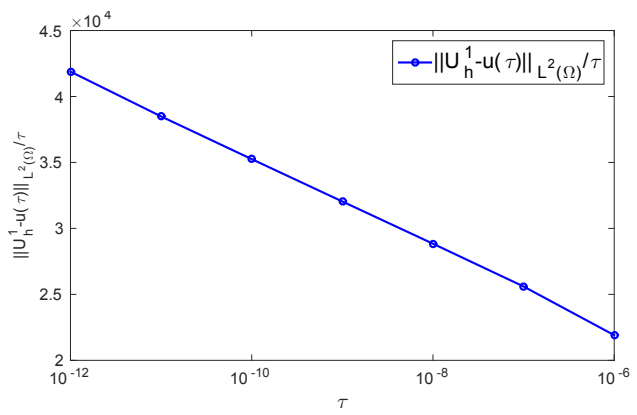

FiguRE 2. The $L^{2}$ errors for (a) at $t_{1}=\tau=10^{-k}, k=$ $5, \ldots, 12$, by convolution quadrature.

\section{Acknowledgements}

The research of B. Jin was partially supported by UK EPSRC Grant EP/M025160/1, that of R. Lazarov and Z. Zhou by NSF Grant DMS1016525, and that of D. Sheen by NRF-2014R1A2A1A11052429.

\section{References}

[1] E. Bazhlekova, Completely monotone functions and some classes of fractional evolution equations. Integral Transforms Spec. Funct. 26, No 9 (2015), 737-752; DOI:10.1080/10652469.2015.1039224.

[2] E. Bazhlekova, B. Jin, R. Lazarov, Z. Zhou, An analysis of the RayleighStokes problem for the generalized second grade fluid. Numer. Math. 131, No 1 (2015), 1-31; DOI:10.1007/s00211-014-0685-2.

[3] A. V. Chechkin, R. Gorenflo, I. M. Sokolov, Retarding subdiffusion and accelerating superdiffusion governed by distributed-order fractional diffusion equations. Phys. Rev. E 66 (2002), 046129; DOI:10.1103/PhysRevE.66.046129 
[4] A. V. Chechkin, R. Gorenflo, I. M. Sokolov, V. Y. Gonchar, Distributed order time fractional diffusion equation. Fract. Calc. Appl. Anal. 6, No 3 (2003), 259-279.

[5] E. Cuesta, C. Lubich, C. Palencia, Convolution quadrature time discretization of fractional diffusion-wave equations. Math. Comp. 75, No 254 (2006), 673-696; DOI: 10.1090/S0025-5718-06-01788-1.

[6] K. Diethelm, N. J. Ford, Numerical analysis for distributed-order differential equations. J. Comput. Appl. Math. 225, No 1 (2009), 96-104; DOI: $10.1016 /$ j.cam.2008.07.018.

[7] N. J. Ford, M. L. Morgado, M. Rebelo, An implicit finite difference approximation for the solution of the diffusion equation with distributed order in time. Electron. Trans. Numer. Anal. 44 (2015), 289-305.

[8] H. Fujita, T. Suzuki, Evolution problems. In: Handbook of Numerical Analysis, Vol. II, North-Holland, Amsterdam (1991), 789-928.

[9] G. Gao, H. Sun, Z. Sun, Some high-order difference schemes for the distributed-order differential equations. J. Comput. Phys. 298, No 1 (2015), 337-359; DOI: 10.1016/j.jcp.2015.05.047.

[10] M. Hasse, The Functional Calculus for Sectorial Operators. Birkhäuser, Basel (2006).

[11] B. Jin, R. Lazarov, Y. Liu, Z. Zhou, The Galerkin finite element method for a multi-term time-fractional diffusion equation. J. Comput. Phys. 281 (2015), 825-843; DOI: 10.1016/j.jcp.2014.10.051.

[12] B. Jin, R. Lazarov, Z. Zhou, Error estimates for a semidiscrete finite element method for fractional order parabolic equations. SIAM J. Numer. Anal. 51, No 1 (2013), 445-466; DOI:10.1137/120873984

[13] B. Jin, R. Lazarov, Z. Zhou, An analysis of the L1 scheme for the subdiffusion equation with nonsmooth data. IMA J. Numer. Anal. 36, No 1 (2016), 197-221; DOI: 10.1093/imanum/dru063.

[14] B. Jin, W. Rundell, A tutorial on inverse problems for anomalous diffusion processes. Inverse Problems 31, No 3 (2015), 035003; DOI: 10.1088/0266-5611/31/3/035003.

[15] J. T. Katsikadelis. Numerical solution of distributed order fractional differential equations. J. Comput. Phys. 259 (2014), 11-22; DOI: 10.1016/j.jcp.2013.11.013.

[16] A. Kilbas, H. Srivastava, J. Trujillo, Theory and Applications of Fractional Differential Equations. Elsevier, Amsterdam (2006).

[17] A. N. Kochubei, Distributed order calculus and equations of ultraslow diffusion. J. Math. Anal. Appl. 340, No 1 (2008), 252-281; DOI: 10.1016/j.jmaa.2007.08.024. 
[18] Z. Li, Y. Luchko, M. Yamamoto, Asymptotic estimates of solutions to initial-boundary-value problems for distributed order time-fractional diffusion equations. Fract. Calc. Appl. Anal. 17, No 4 (2014), 1114-1136; DOI: 10.2478/s13540-014-0217-x; http://www.degruyter.com/view/j/

fca.2014.17.issue-4/issue-files/fca.2014.17.issue-4.xml.

[19] C. Lubich, Convolution quadrature and discretized operational calculus, I. Numer. Math. 52, No 2 (1988), 129-145; DOI: 10.1007/BF01398686.

[20] C. Lubich, I. H. Sloan, V. Thomée, Nonsmooth data error estimates for approximations of an evolution equation with a positive-type memory term. Math. Comp. 65, No 213 (1996), 1-17; DOI: 10.1090/S0025-571896-00677-1.

[21] Y. Luchko, Boundary value problems for the generalized time fractional diffusion equation of distributed order. Fract. Calc. Appl. Anal., 12, No 4 (2009), 409-422; at http://www.math.bas.bg/ fcaa.

[22] F. Mainardi, A. Mura, G. Pagnini, R. Gorenflo, Time-fractional diffusion of distributed order. J. Vibr. Control 14, No 9-10 (2008), 12671290; DOI: $10.1177 / 1077546307087452$.

[23] E. Martensen, Zur numerischen Auswertung uneigenlicher Integrale. Z. Angew. Math. Mech. 48 (1968), T83-T85.

[24] W. McLean, V. Thomée, Numerical solution via Laplace transforms of a fractional order evolution equation. J. Integral Equations Appl. 22, No 1 (2010), 57-94; DOI: 10.1216/JIE-2010-22-1-57.

[25] M. M. Meerschaert, H.-P. Scheffler, Stochastic model for ultraslow diffusion. Stochastic Process. Appl. 116, No 9 (2006), 1215-1235; DOI: 10.1016/j.spa.2006.01.006.

[26] M. L. Morgado, M. Rebelo, Numerical approximation of distributed order reaction diffusion equations. J. Comput. Appl. Math. 275 (2015), 216-227; DOI: 10.1016/j.cam.2014.07.029.

[27] D. Sheen, I. Sloan, V. Thomée, A parallel method for timediscretization of parabolic problems based on contour integral representation and quadrature. Math. Comp. 69, No 229 (2000), 177-195; DOI: 10.1090/S0025-5718-99-01098-4.

[28] D. Sheen, I. Sloan, V. Thomée, A parallel method for time discretization of parabolic equations based on Laplace transformation and quadrature. IMA J. Numer. Anal. 23, No 2 (2003), 269-299; DOI: 10.1093/imanum/23.2.269.

[29] I. M. Sokolov, A. V. Chechkin, J. Klafter, Distributed-order fractional kinetics. Acta Phys. Polon. B 35, No 4 (2004), 1323-1341. 
[30] V. Thomée, Galerkin Finite Element Methods for Parabolic Problems. Springer-Verlag, Berlin (2006).

[31] S. Umarov, Continuous time random walk models associated with distributed order diffusion equations. Fract. Calc. Appl. Anal. 18, No 3 (2015), 821-837; DOI: 10.1515/fca-2015-0049; http://www.degruyter.com/view/j/

fca.2015.18.issue-3/issue-files/fca.2015.18.issue-3.xml.

[32] J. A. C. Weideman, L. N. Trefethen, Parabolic and hyperbolic contours for computing the Bromwich integral. Math. Comp. 76, No 259 (2007), 1341-1356; DOI: 10.1090/S0025-5718-07-01945-X.

${ }^{1}$ Dept. of Computer Science, University College London

Gower Street, London WC1E 6BT, UK

e-mail: bangti.jin@gmail.com,b.jin@ucl.ac.uk

2 Dept. of Mathematics, Texas A\&M University

College Station, TX 77843-3368, USA

e-mail:lazarov@math.tamu.edu

Received: June 5, 2015

3 Dept. of Mathematics, Seoul National University

Seoul 151-747, KOREA

e-mail:dongwoosheen@gmail.com

${ }^{4}$ Dept. of Applied Physics and Applied Mathematics

Columbia University

New York, NY, 10027, USA

e-mail: zhizhou0125@gmail.com

Please cite to this paper as published in:

Fract. Calc. Appl. Anal., Vol. 19, No 1 (2016), pp. 69-93, DOI: $10.1515 /$ fca-2016-0005 